\title{
Digalactosyldiacylglycerol Synthase Gene MtDGD1 Plays an Essential Role in Nodule Development and Nitrogen Fixation
}

\author{
Zaiyong Si, Qianqian Yang, Rongrong Liang, Ling Chen, Dasong Chen, and Youguo $\mathrm{Li}^{+}$ \\ State Key Laboratory of Agricultural Microbiology, Huazhong Agricultural University, Wuhan 430070, People's Republic of China \\ Accepted 13 April 2019.
}

Little is known about the genes participating in digalactosyldiacylglycerol (DGDG) synthesis during nodule symbiosis. Here, we identified full-length MtDGD1, a synthase of DGDG, and characterized its effect on symbiotic nitrogen fixation in Medicago truncatula. Immunofluorescence and immunoelectron microscopy showed that MtDGD1 was located on the symbiosome membranes in the infected cells. $\beta$-Glucuronidase histochemical staining revealed that MtDGD1 was highly expressed in the infection zone of young nodules as well as in the whole mature nodules. Compared with the control, MtDGD1RNA interference transgenic plants exhibited significant decreases in nodule number, symbiotic nitrogen fixation activity, and DGDG abundance in the nodules, as well as abnormal nodule and symbiosome development. Overexpression of $M t D G D 1$ resulted in enhancement of nodule number and nitrogen fixation activity. In response to phosphorus starvation, the MtDGD1 expression level was substantially upregulated and the abundance of nonphospholipid DGDG was significantly increased in the roots and nodules, accompanied by corresponding decreases in the abundance of phospholipids such as phosphatidylcholine, phosphatidylethanolamine, and phosphatidylinositol. Overall, our results indicate that $D G D 1$ contributes to effective nodule organogenesis and nitrogen fixation by affecting the synthesis and content of DGDG during symbiosis.

Keywords: DGDG, Medicago truncatula, MtDGD1, phospholipids, rhizobium-legume symbiosis, RNAi, symbiotic phenotype

Lipids, including phospholipids and glycolipids, are the main constituents of membranes and play key roles in the biological function processes of membranes. In many higher animals and yeast cells, phospholipids such as phosphatidylcholine (PC), phosphatidylethanolamine (PE), and phosphatidylinositol (PI) are the main components of membrane lipids. However, in plant

${ }^{\dagger}$ Corresponding author: Y. Li; youguoli@mail.hzau.edu.cn

Funding: This work was supported by the funds from the National Key Research and Development Program of China (grant number 2016YFD0100700), the National Natural Science Foundation of China (grant numbers 31670243 and 31371549), and the Fundamental Research Funds for the Central Universities (2016PY025).

*The $\boldsymbol{e}$-Xtra logo stands for "electronic extra" and indicates that six supplementary figures and three supplementary tables are published online.

The author(s) declare no conflict of interest.

๑) 2019 The American Phytopathological Society leaf cells, the contents of glycolipids, particularly galactolipids, are much higher than those of phospholipids (Dörmann and Benning 2002). Galactolipids monogalactosyldiacylglycerol (MGDG) and digalactosyldiacylglycerol (DGDG), the two most common nonphospholipids, account for approximately $80 \%$ of the total membrane lipids in plant cells, and are also the main components of chloroplast thylakoid membranes (Dörmann and Benning 2002). It has been reported that MGDG and DGDG are enriched in thylakoids to maintain their stability (Krumova et al. 2010; Shimojima and Ohta 2011), the functional intactness of the complexes of photosystems I and II (Härtel et al. 1997; Reifarth et al. 1997), and the thermosensitivity of the photosynthetic apparatus (Essemine et al. 2011). Interestingly, the nonredundant function of MGDG and DGDG was found in systemic acquired resistance in plants, and DGDG was reported to be essential for pathogen-responsive nitric oxide and salicylic acid accumulation (Gao et al. 2014). MGDG can be converted into DGDG and oligogalactolipids under extreme conditions, and the generated DGDG would accumulate on the extraplastidic membranes to resist stressful environments (Slack et al. 1977). The symbiosis establishment of fungi and bacteria-namely, Rhizopus microsporus (Mucoromycotina) and endobacteria in genus Burkholderia-was found to be accompanied by specific changes in the fungal lipid profile. Diacylglycerol kinase (DGK) activity is critical for these lipid metabolic changes when DGK inhibition alters the fungal lipid profile, resulting in a shift from the host-bacteria interaction to antagonism (Lastovetsky et al. 2016). All of these studies indicate the importance of host lipid remodeling in the interaction between the eukaryote and bacteria or the environment.

Nodule formation and development involve mutualistic interactions between legume plants and rhizobia and are essential for symbiotic nitrogen fixation (Oldroyd et al. 2011). In response to the symbiotic signal from the roots of plants, the rhizobia invade root hairs through infection threads (IT), and eventually reach cortical cells and enter infected plant cells, where each rhizobium cell is wrapped by a membrane of plant origin and is separated into a compartment (Jones et al. 2007). The membrane is formed by invagination of the plasma membrane of plant cells, in which the rhizobia are deposited in a process resembling endocytosis. These compartments, consisting of one or more bacteroids surrounded by a plant-derived membrane, are called symbiosomes. The membrane of symbiosomes is also called the peribacteroid membrane (PBM), which serves as the boundary of plant cell cytoplasm and bacteroids. The symbiosome responsible for symbiotic nitrogen fixation is a functional unit in nodules. Symbiosome membranes regulate the exchanges of ammonium, nutrients, and signaling molecules between the bacteroids and host plants (Suzaki and Kawaguchi 2014). The function of the symbiosome 
membrane in symbiotic nitrogen fixation depends on its specific composition of proteins and lipids. The symbiosome membrane is both the structural and functional interface between plant cells and bacterial cells.

Notably, DGDG was also detected in the roots, leaves, symbiosomes, and symbiosome membranes of soybean (Glycine max) and Lotus japonicus but is not present in bacteroids and free-living rhizobial cells, suggesting that DGDG is a type of host plant-synthesized lipid which is transported into the symbiosome. Accumulated DGDG in nodules and symbiosome membranes might help to save phosphorus for effective legumerhizobium symbiosis (Gaude et al. 2004). In another study, Bradyrhizobium and soybean nodules grown in nitrogen-deficient soil showed dramatic increases in the levels of MGDG and DGDG in the leaves (Narasimhan et al. 2013). However, there have been no reports about the variation of DGDG in Medicago truncatula in response to environmental stress. Lipids in the host-derived membrane are considered to be synthesized in the endoplasmic reticulum and eventually transported to the PBM through the Golgi apparatus (Brewin 1990). In Chinese milk vetch (Astragalus sinicus), a nodule-specific lipid transfer protein, AsE246, which is specifically expressed in nodules, could bind the plant-synthesized membrane lipid DGDG in vivo. Furthermore, the knockout of AsE246 not only reduced the formation of infection threads and nodule primordia but also decreased the content of lipids, particularly that of DGDG in the nodules, suggesting that AsE246 is involved in the transport of lipids into the symbiosome membrane. In addition, it was confirmed that DGDG was colocalized with the bacteroids and was localized on the symbiosome membranes (Lei et al. 2014). All of these studies indicate that symbiotic nitrogen fixation has important effects on membrane lipid remodeling and DGDG biosynthesis plays an important role in nodule development and symbiotic nitrogen fixation. However, these effects have not been fully characterized yet.

The biosynthesis of DGDG in Arabidopsis thaliana was reported to be involved in the galactosylation of MGDG by the galactolipid:galactolipid galactosyltransferase (GGGT), and three genes, including digalactosyldiacylglycerol synthase 1 (DGD1), DGD2 and GGGT, are known to encode DGDG synthases (Benning and Ohta 2005). The $d g d 1$ mutant of A. thaliana showed a $90 \%$ decrease in the content of DGDG and severely suppressed growth. However, there was little difference in phenotype between the $d g d 2-1$ mutant and the wild type (WT) (Kelly et al. 2003). In addition, DGD2 was expressed at low levels under normal conditions but strongly induced under phosphorus deprivation conditions (Kobayashi et al. 2009). Reverse genetic analysis showed that $G G G T$ is a cold-sensitive gene in A. thaliana, indicating that GGGT is not essential for the normal growth and development of plants (Moellering et al. 2010). These previous findings suggest that $D G D 1$ is critical for the biosynthesis of the bulk amount of DGDG in the chloroplasts (Kelly and Dörmann 2002). It is generally known that $D G D 1$ is mainly responsible for the biosynthesis of DGDG in plant cells under normal conditions.

As described above, a large amount of DGDG needs to be synthesized by the host plants to facilitate rhizobial infection, symbiosome development, and nitrogen fixation; however, the biological function of DGDG in the nodules of legume plants is not fully understood. The symbiosis phenotypes have not been characterized when DGDG synthesis is affected. In particular, little is known about the functions of those genes involved in DGDG biosynthesis in symbiotic interactions between legume plants and rhizobia. In addition, $M$. truncatula forms indeterminate-type nodules and is different from soybean and L. japonicas, which form determinate-type nodules. Hence, it is highly essential to clarify the function of DGDG in symbiotic nitrogen fixation in $M$. truncatula. In addition, there have been few reports about the changes in the biosynthesis and abundance of DGDG in response to low phosphorus stress during legume-rhizobia symbiosis under nitrogen-deficient conditions.

This study identified $M t D G D 1$, a synthase of DGDG, and characterized its effect on the symbiotic nitrogen fixation in $M$. truncatula through a series of experiments, including promoter cloning, in situ expression pattern analysis, protein subcellular localization, symbiotic phenotype analysis of transgenic plants with gene silencing or overexpression, and determination of changes in DGDG accumulation in response to low phosphorus stress. Our results are expected to clarify the relationship between the MtDGDl gene related to DGDG biosynthesis and DGDG accumulation in nodules as well as the effect of the MtDGDl gene on nodulation and nitrogen fixation. Our findings provide novel insights into the membrane lipid remodeling in root nodule development and nitrogen fixation.

\section{RESULTS}

\section{Cloning and phylogenetic characterization of MtDGD1 gene.}

We performed a blast analysis based on the query sequences available in a previous publication (Gaude et al. 2004), including AY635907 (Gm-DGD1), AY635908 (Gm-DGD2), Y635909 (LjDGD1), and AY635910 (Lj-DGD2). The results showed that, in the $M$. truncatula genome, there were two DGD1-namely, XM_013594759.2 (Medtr7g106250.1) and XM_013612439.2 (Medtr1g057700.1) — and four DGD2—namely, XM_024775447.1 (Medtr1g047640.2), XM_013611780.2 (Medtr1g047640.1), XM 003620822.3 (Medtr6g091670.1), and XM_003623811.3 (Medtr7g076400.1). The transcriptome data (Young et al. 2011) indicated that the transcript levels of XM_013594759.2 and XM_003620822.3 were the highest in the nodule (Supplementary Fig. S1), which were therefore chosen for further analysis and named MtDGD1 and MtDGD2, respectively.

Based on the data from phytozome v11.0, PCR primers were designed to clone the gene MtDGDl (accession number XM_013594759.2). The full-length cDNA of MtDGD1 was obtained, which contained an open reading frame of 2,343 bp that encodes a protein of 780 amino acid (aa) residues with a putative molecular weight of approximately $88 \mathrm{kDa}$. The full-length cDNA of MtDGD2 (accession numberXM_003620822.3) contained an open reading frame of $1,392 \mathrm{bp}$ that encodes a protein of 464 aa residues with a putative molecular weight of approximately $53 \mathrm{kDa}$. Conserved domain analysis showed that the proteins MtDGD1 (accession number XP_013450213.1) and MtDGD2 (accession number XP_003620870.1) belonged to the glycosyltransferase GTB-type superfamily (Supplementary Fig. S2). MtDGD1 and MtDGD2 had a glycosyltransferase GTB-type superfamily domain at the positions of 535 to 670 and 1 to 464 aa, respectively, in the $\mathrm{C}$ terminal. Amino acid sequence alignment of MtDGD1 and another DGD1 (XP_013467893.1) indicated that MtDGD1 was longer than XP_013467893.1 in the N terminal, and they had a high degree of homology at the $\mathrm{C}$ terminal. Amino acid sequence alignment of MtDGD2 and three other DGD2 proteins (XP_024631215.1, XP_013467234.1, and XP_003623859.1) indicated that these four DGD2 proteins had almost identical amino acid sequences. In addition, the amino acid sequence of MtDGD1 was compared with that of DGD1 in other higher plants, including leguminous plants. The results revealed that MtDGD1 had 91, 88, and $87 \%$ similarities in amino acid sequence to the DGD1 in Cicer arietinum, L. japonicus, and soybean (G. max), respectively (Supplementary Table S1) but only a 71\% similarity to the DGD1 in Arabidopsis. In addition, a maximum-likelihood phylogenetic analysis of these DGD1 proteins was performed using MEGA6.0 software. The phylogenetic tree (Fig. 1) indicated that DGD1 


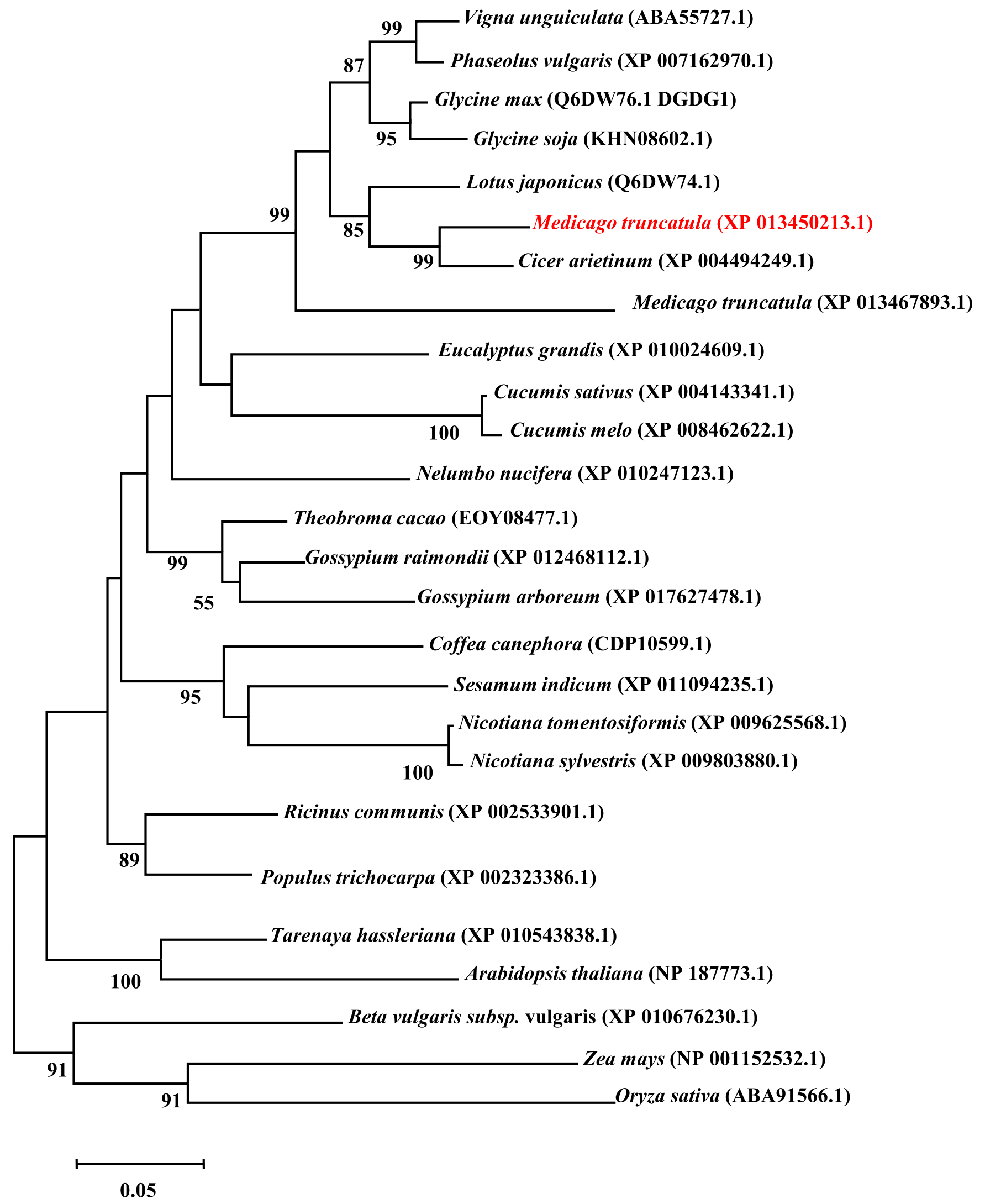

Fig. 1. Phylogenetic analysis of the MtDGD1 protein. BLASTP of MtDGD1 and its homologs from phytozome v11.0 and NCBI was performed. Accession numbers of the proteins are indicated in the subsequent parentheses. The maximum-likelihood method was used for the phylogenetic analysis. Evolutionary analyses were performed by MEGA6.0 software. Bootstrap values were from 1,000 replications. 
proteins are widely present in higher plants, including leguminous plants.

\section{Expression and localization of $M t D G D 1$ protein during symbiosis.}

To determine the subcellular location of MtDGD1 under symbiotic conditions, an immunofluorescence analysis of WT M. truncatula inoculated with green fluorescent protein (GFP)labeled Sinorhizobium meliloti 1021 was carried out. The antiMtDGD1 antibody was used and its specificity was confirmed via Western blotting (Supplementary Fig. S3). The nodules were harvested at 35 days postinoculation (dpi). The localization of MtDGD1 was observed by a laser confocal microscopy. The observation showed a substantial overlap of CY3-red and GFP-green fluorescence in the nodules and infected cells (Fig. $2 \mathrm{C}$ and 2D), and no red fluorescence was found in the negative nodule slides without the addition of MtDGD1 antibodies (Fig. 2A) or with the addition of preimmune sera (Fig. 2B). These results indicated that MtDGD1 was colocalized with bacteroids in the infected cells. Considering that DGDG is a plant-derived lipid and accumulates in the PBM (Gaude et al. 2004), it can be hypothesized that MtDGD1 may be localized on the symbiosome membrane. Immunoelectron microscopy was implemented to determine the subcellular localization of MtDGD1 during symbiosis. The results showed that MtDGD1 was located on the symbiosome membrane (Fig. $3 \mathrm{C}$ to $\mathrm{F}$ ), and no immunogold signal was observed in the negative control without the addition of MtDGD1 antibody (Fig. 3A) or with the addition of preimmune sera (Fig. 3B). All of these results indicated that MtDGD1 is localized on the symbiosome membrane. In addition, the immune

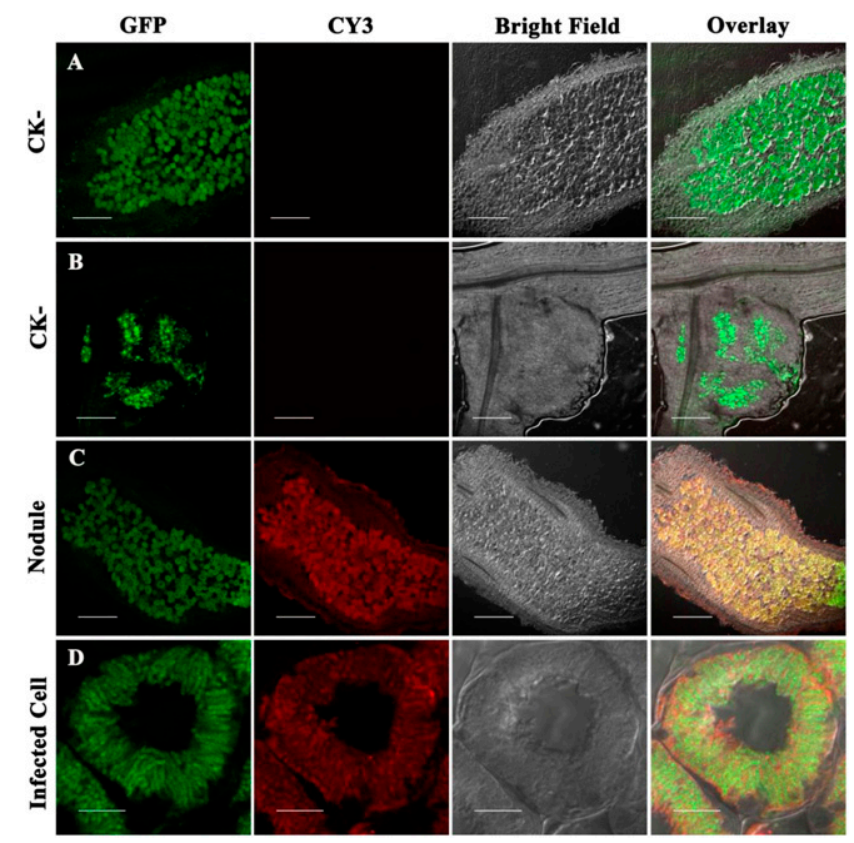

Fig. 2. Immunolocalization of MtDGD1 in the nodules of Medicago truncatula. Different signal dots indicate green fluorescent protein (GFP)labeled Sinorhizobium meliloti 1021 or protein MtDGD1. Secondary antibody is tagged with CY3. A and B, Negative control (CK-); $\mathbf{A}$, without the addition of MtDGD1 antibody and $\mathbf{B}$, with the addition of the preimmune sera. Other treatments were the same as those in the experimental group. No red fluorescence CY3 signal was detected. C, Immunolocalization of MtDGD1 in the root nodules. The signal was only observed in the infected cells. D, Immunolocalization of MtDGD1 in infected cells. The signal was observed to be specifically colocalized with symbiosomes in root nodules. Bars $=250 \mu \mathrm{m}(\mathrm{A}, \mathrm{B}$, and C) and $10 \mu \mathrm{m}(\mathrm{D})$. localization of MtDGD1 in the roots of M. truncatula was examined, and the results showed that MtDGD1 was located in the root hair cells and the membrane of root cells (Supplementary Fig. S4).

\section{Expression pattern of $M t D G D 1$}

during nodule development.

The gene expression profiles in $M$. truncatula revealed a higher transcript level of MtDGD1 in nodules than in roots, as well as in the nitrogen-fixation zone (zone III) than in other zones of the whole nodule (Supplementary Fig. S5). To validate this result, real-time PCR was performed to examine the spatial transcription of MtDGD1 in different tissues inoculated or uninoculated with rhizobia. The transcript levels of $M t D G D 1$ in the stems, leaves, and roots inoculated with S. meliloti 1021 were upregulated compared with those of the control group uninoculated with rhizobia, and the plants watered with nitrogen-free Fahraeus nutrient solution (NFS). The highest transcript level of MtDGD1 was observed in the leaves, followed by the nodules (Fig. 4A). To further determine whether $M t D G D 1$ is involved in nodule development or nitrogen fixation, the temporal expression pattern of $M t D G D l$ was also examined. The results showed that the transcript levels of $M t D G D 1$ in the roots and nodules increased gradually at $5 \mathrm{dpi}$ and reached the maximal level at 15 dpi (Fig. 4B). In addition, we analyzed the gene expression pattern of MtCOPT1 (XM_003608827), the copper transporter 1 of M. truncatula,
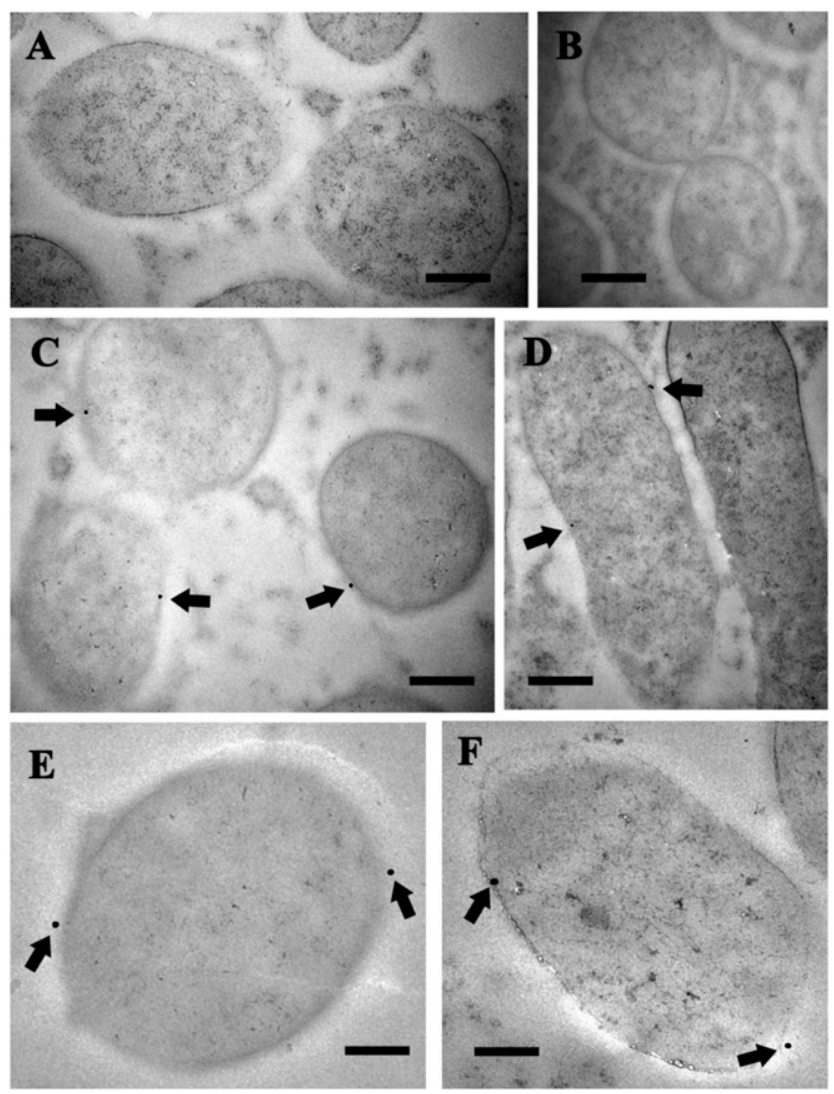

Fig. 3. Ultrastructural localization of MtDGD1 on the symbiosome membranes in root nodules. A, Negative control without the addition of MtDGD1 antibody. B, Negative control with addition of the preimmune sera as the first antibody. C, D, E, and F, Immunogold signals were detected (but not in A and B). The 10-nm gold particles were present on the symbiosome membranes (immunogold signal is marked as black dots, indicated by black arrowheads). Bars $=300 \mathrm{~nm}$ (A and C), $500 \mathrm{~nm}$ (B and D), and 200 $\mathrm{nm}$ (E and F). 
whose expression is known to be specific to nodules (Senovilla et al. 2018). The results showed that MtCOPT1 was only expressed in root nodules, and reached the highest level at 15 dpi (Fig. 4C). In addition, the transcript levels of housekeeping gene MtEF1- $\alpha$ (XM_013595882.2) were also checked. The results showed that the expression of MtEF1- $\alpha$ was rather stable, without remarkable changes in the process of nodule development (Fig. 4D). These findings indicated that MtDGDI is involved in nodule development or nitrogen fixation.

To further analyze the spatial expression pattern of the $M t D G D 1$ gene during nodule development, transformation of M. truncatula was carried out. M. truncatula transfected with a plasmid pDX2181G-mcherry containing a fusion of the MtDGD1 promoter (a 2.4-kb fragment upstream of the MtDGD1 ATG codon) served as the treatment group, while M. truncatula transfected with an empty plasmid pDX2181Gmcherry was used as the control. The transgenic roots were confirmed and then inoculated with $S$. meliloti 1021. Histochemical $\beta$-glucuronidase (GUS) staining was performed to analyze the spatial distribution of the GUS activity (Sessions et al. 1999). As a result, GUS activity was detected in the vascular cylinder and the tip of transgenic roots (Fig. 5B), as well as in the nodule primordia at 5 dpi (Fig. 5C). It was noteworthy that $M t D G D 1$ was highly expressed in the infection zone of young nodules (Fig. 5E). In 15-dpi nodules, GUS activity was observed in the cells in the infection zone but not found in the cells in the nitrogen fixation zone (Fig. 5E and F). However, GUS activity was detected in the infection zone as well as in the entire nodule at 21 and 32 dpi (Fig. 5H, I, K, and L). No GUS activity was detected in the control group (Fig. 5A, $\mathrm{D}, \mathrm{G}$, and J). Both histochemical GUS and real-time PCR analysis showed the highest expression level of MtDGDl in the nodules. These results demonstrated a dynamic expression pattern of MtDGD1 during nodule development, suggesting that $M t D G D 1$ affects nodule development and symbiotic nitrogen fixation.

\section{Overexpression of $M T D G D 1$ in hairy roots increased the nodule number.}

Transgenic $M$. truncatula plants overexpressing MtDGD1 were generated by transformation utilizing Agrobacterium rhizogenes MSU440 and confirmed by GUS staining. Nodules were collected at $28 \mathrm{dpi}$, and the transcript levels of MtDGD1 were examined by real-time PCR. The transcript level of
A

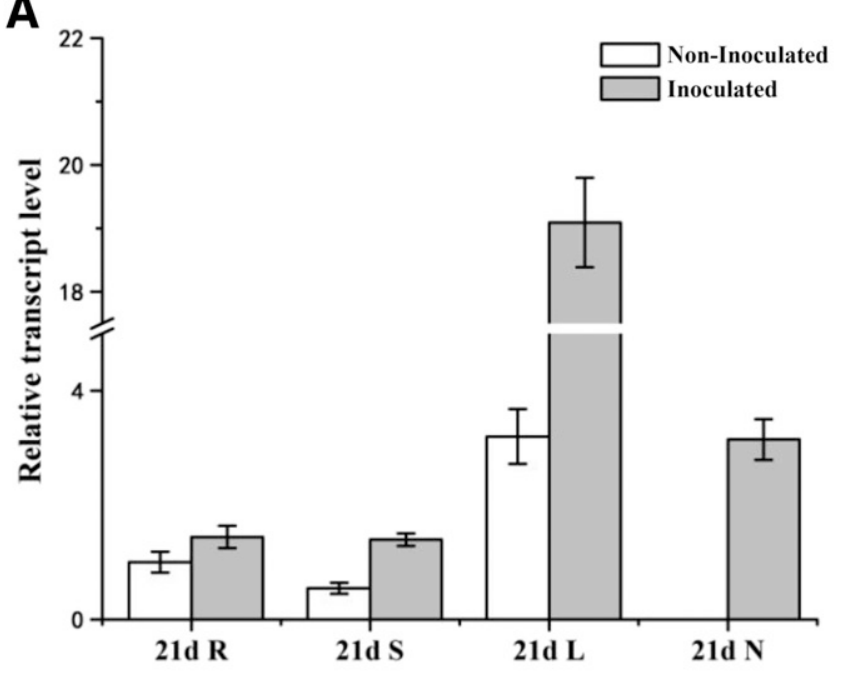

C

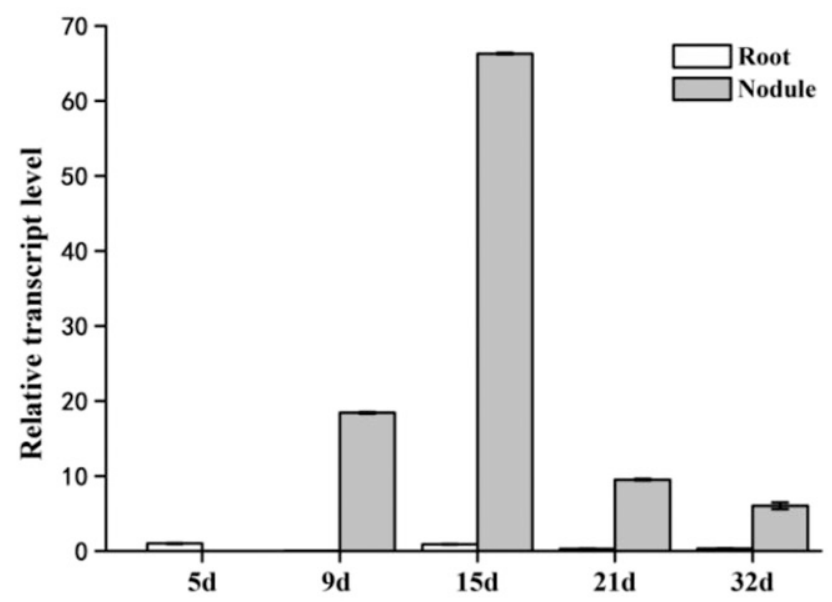

B

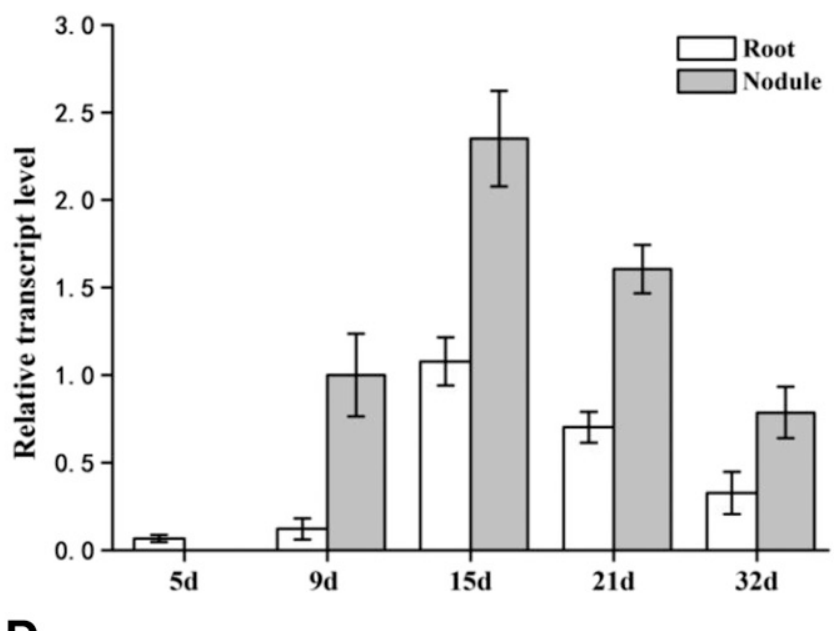

D

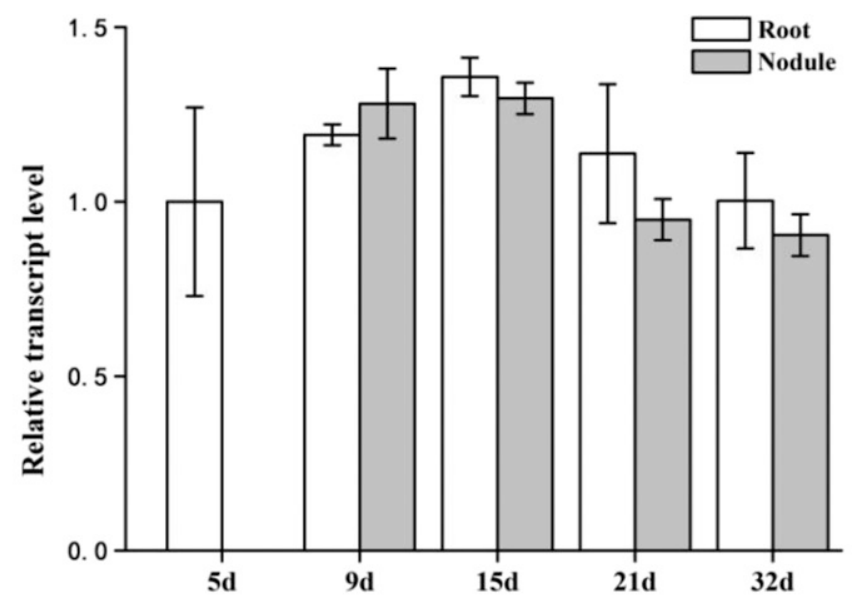

Fig. 4. Expression patterns of MtDGD1 under symbiosis in Medicago truncatula. A, Tissue-specific transcription of MtDGD1 in the roots (R), stems (S), leaves $(\mathrm{L})$, and nodules $(\mathrm{N})$ at 21 days postinoculation (dpi). Noninoculated plants watered with nitrogen-free Fahraeus nutrient solution served as the control. B, Transcription of MtDGD1 in the roots and nodules at 5, 9, 15, 21, and 32 dpi. C, Transcription of MtCOPT1 in the roots and nodules at 5, 9, 15, 21, and 32 dpi. D, Transcription of MtEF1- $\alpha$, a housekeeping gene in the roots or nodules at 5, 9, 15, 21, and 32 dpi. All of the transcript levels were determined using GAPDH as the internal standard gene. Error bars represent the standard deviations of three independent experiments. 
$M t D G D 1$ in the transgenic nodules was 38 -fold that in the control nodules transfected with the empty vector pCAMBIA 2301 (Supplementary Fig. S6). Liquid chromatography-mass spectrometry (LC-MS) analysis was used to measure the abundance of DGDG in MtDGDl-overexpressing transgenic nodules. The results showed slightly higher abundance of DGDG in transgenic nodules than in the control (Supplementary Table S3). Additionally, symbiotic phenotypes were examined at 28 dpi. The results showed that the nitrogenase activity of the transgenic nodules was slightly higher than that of the control, and the average number of nodules on the transgenic roots was 23.78 per hairy root $(n=20)$, which was significantly higher than that on the control roots (17.6 per hairy root, $n=20$ ) (Table 1 ).

MtDGD1 RNA interference-impaired nodule development and nitrogen fixation.

To address the functional phenotypes of MtDGD1 in nodule development, RNA interference (RNAi) was used to suppress the expression of MtDGDl. Real-time PCR showed that the RNAi efficiency of MtDGDl in transgenic nodules was approximately $70 \%$ (Fig. 6D) lower than that in the control nodules

\section{Control}
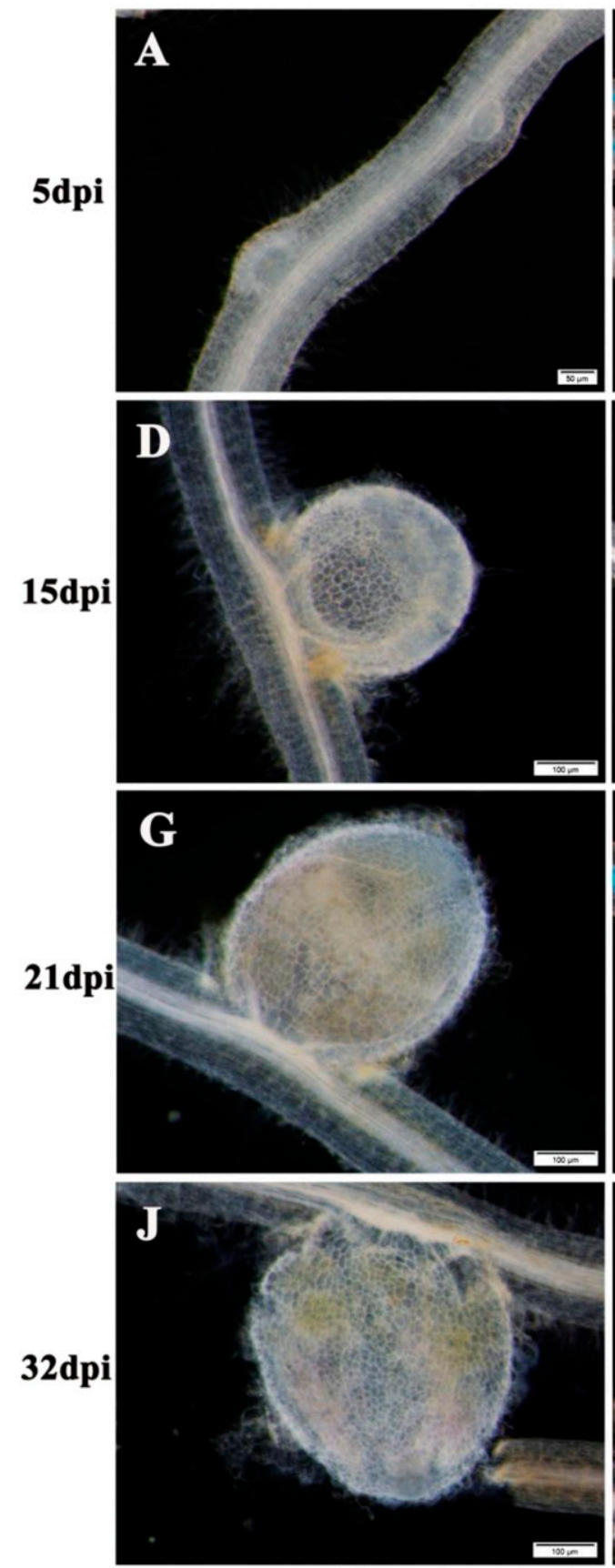

MtDGD1prom:GUS
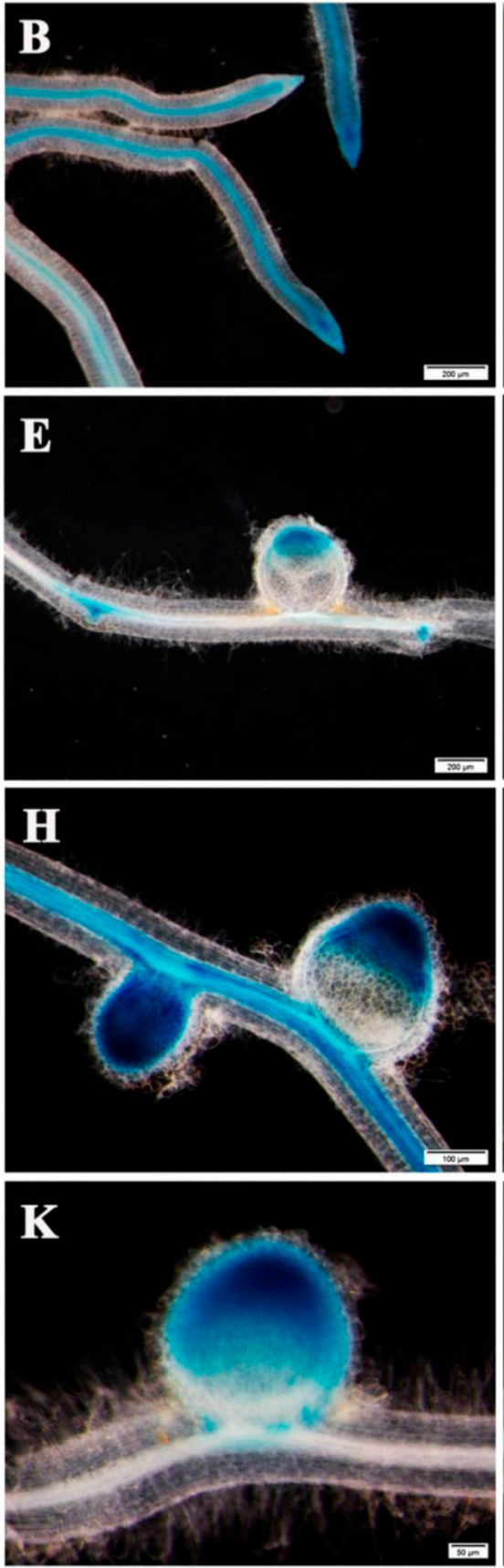

MtDGD1prom:GUS
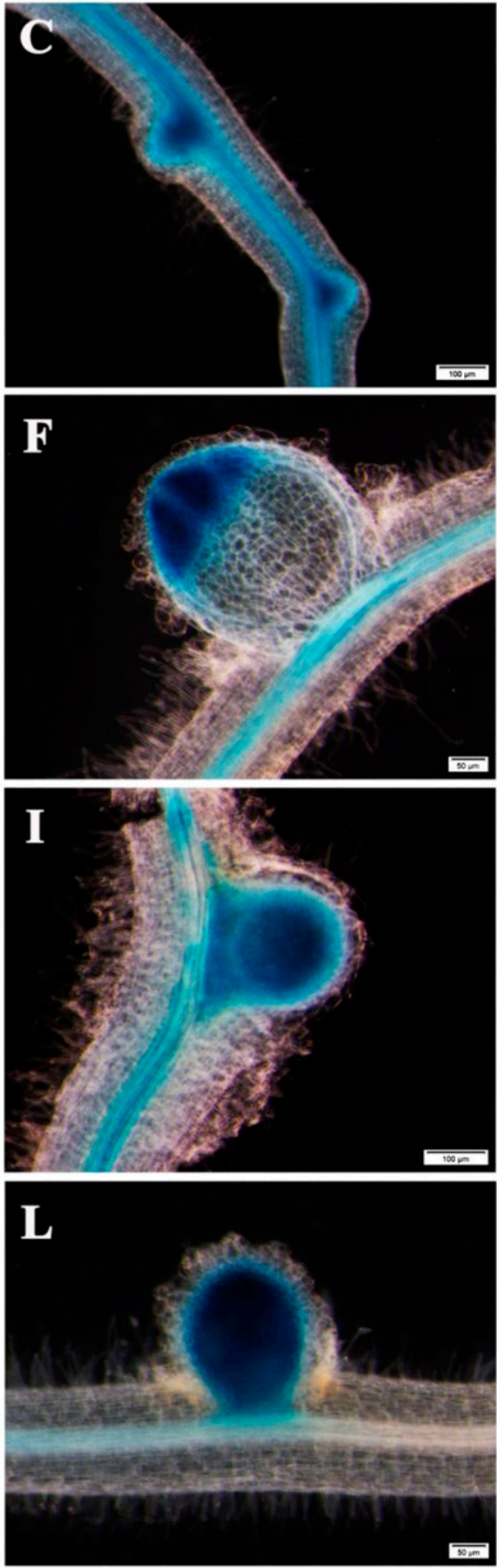

Fig. 5. Temporal and spatial in situ expression of $M t D G D 1$ during symbiotic conditions. A, D, G, and J, Control group with empty plasmid DX2181G-mcherry. $\mathbf{B}$ and $\mathbf{C}$, Transgenic plants in $\beta$-glucuronidase (GUS) staining at 5 days postinoculation (dpi); $\mathbf{E}$ and $\mathbf{F}$, transgenic plants in GUS staining at 15 dpi; $\mathbf{H}$ and $\mathbf{I}$, transgenic plants in GUS staining at $21 \mathrm{dpi} ; \mathbf{K}$ and $\mathbf{L}$, transgenic plants in GUS staining at 32 dpi. Bars $=500 \mu \mathrm{m}(\mathrm{A}, \mathrm{F}, \mathrm{K}$, and L), $100 \mu \mathrm{m}(\mathrm{C}, \mathrm{D}, \mathrm{G}, \mathrm{H}, \mathrm{I}$, and J), and $200 \mu \mathrm{m}$ (B and $\mathrm{E})$. 
transfected with the empty vector pCAMBIA1301-35S-int-T7. We also checked the transcript level of $M t E F 1-\alpha$ in the transgenic RNAi nodules. The results indicated that the transcript level of MtEF1- $\alpha$ was unchanged (Fig. 6D). In addition, we noted that MtDGDI-RNAi did not suppress the transcription of $M t D G D 2$ in the nodules. Conversely, the transcript level of $M t D G D 2$ was increased compared with that in the control nodules (Fig. 6D). The control plantlets displayed vigorous growth, with green leaves and red nodules, whereas the RNAi transgenic plantlets were small, with chlorotic leaves and white, small nodules (Fig. 6A to C). Symbiotic phenotypes were further examined and quantified at $28 \mathrm{dpi}$. The results showed that the fresh weight of the aerial parts of the RNAi transgenic plantlets was significantly lower than that of the control (Table 1). Moreover, the nodule nitrogenase activity $\left(9.51 \mu \mathrm{mol}\right.$ g nodul $\left.\mathrm{e}^{-1} \mathrm{~h}^{-1}, n=9\right)$ and number of root nodules $(10.4, n=20)$ per hairy root on the RNAi hairy roots were significantly decreased compared with those in the control (Table 1).

To further clarify the influence of MtDGDl RNAi on nodule formation and development, paraffin sections of MtDGD1-RNAi nodules at 28 dpi were observed. Significantly fewer infected cells and rhizobia were observed in MtDGD1-RNAi nodules, and most infected cells were localized in the infection zone (Fig. 7B and D). Few infected cells were found in the nitrogen fixation zone of the nodules. The infected cells displayed deformed morphologies with indistinct boundaries compared with the control (Fig. 7A to D). In addition, ultrastructural comparison of the MtDGD1-RNAi

Table 1. Symbiotic phenotypes of $M t D G D 1-$ RNAi and over-expression transgenic plants ${ }^{\mathrm{a}}$

\begin{tabular}{|c|c|c|c|}
\hline Group & $\begin{array}{l}\text { Nodule nitrogenase activity } \\
\left(\mu \mathrm{mol} g \text { nodule }{ }^{-1} \mathbf{h}^{-1}\right)\end{array}$ & Shoot fresh weight (g/hairy plant) & Nodule number (per hairy root) \\
\hline MtDGD1-CK & $23.90 \pm 4.03$ & $0.35 \pm 0.13$ & $17.6 \pm 5.36$ \\
\hline MtDGD1-RNAi & $9.51 \pm 1.50 *$ & $0.06 \pm 0.015^{*}$ & $10.4 \pm 2.8^{*}$ \\
\hline MtDGD1-OX & $25.64 \pm 2.76$ & $0.37 \pm 0.16$ & $23.78 \pm 4.8 *$ \\
\hline
\end{tabular}

a Symbiotic phenotypes (nodule nitrogenase activity, shoot fresh weight, and nodule numbers per hairy root) were examined at 28 days postinoculation. MtDGD1-CK was the transgenic plants transfected with the empty vector. MtDGD1-RNAi and MtDGD1-OX were transgenic plants transfected with the RNA interference and the overexpression vector, respectively. Asterisks (*) indicate significant differences between the two groups at the level of $P<0.05$. The data represent three independent experiments $(n=20)$.
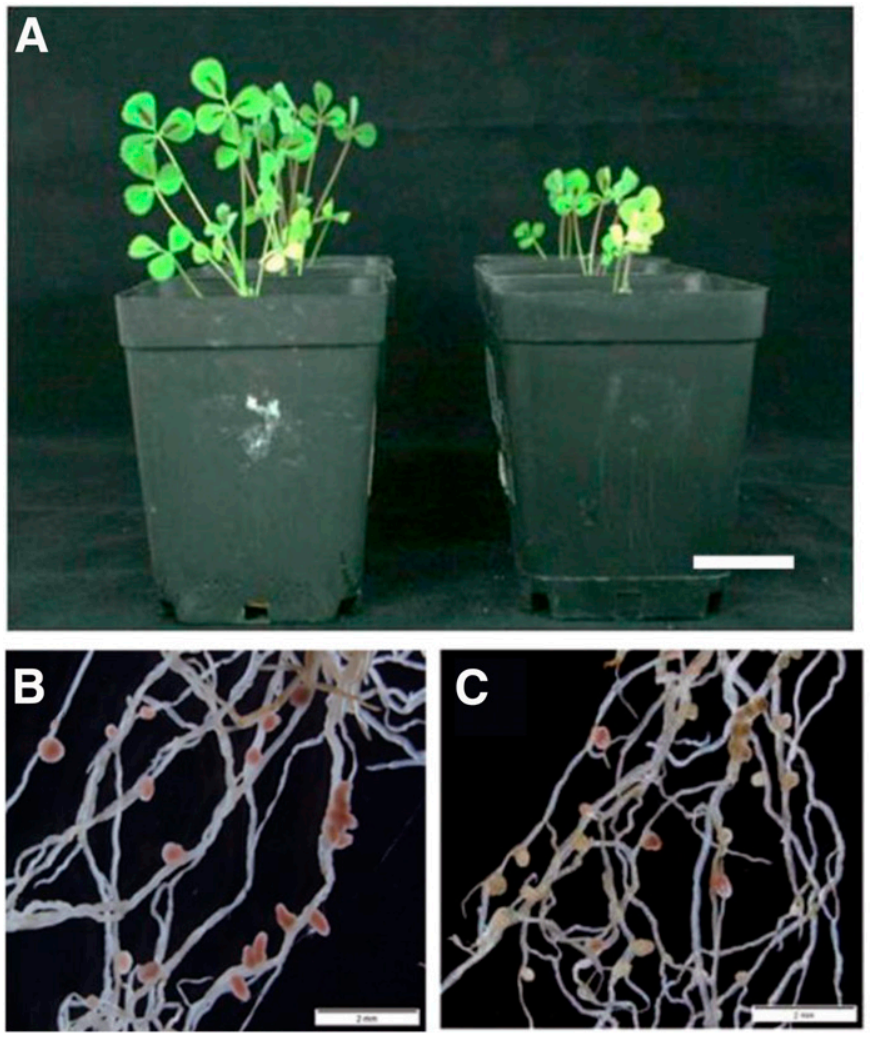
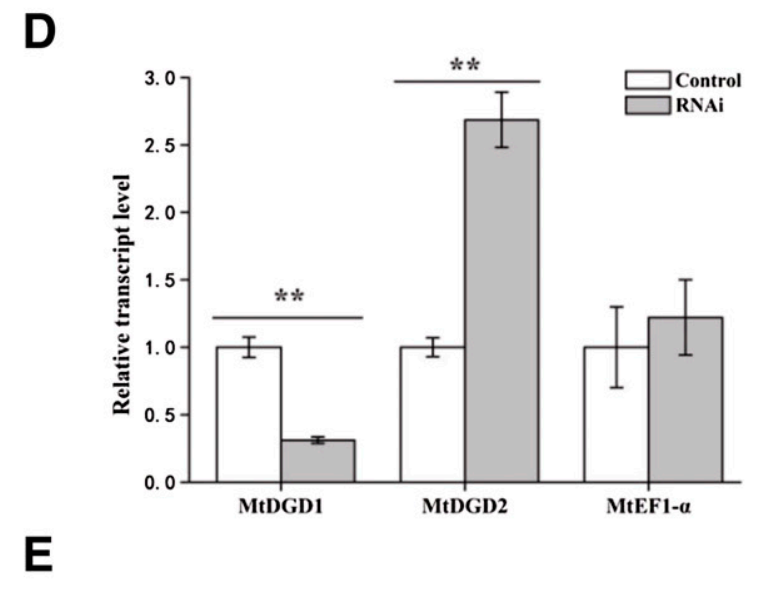

E

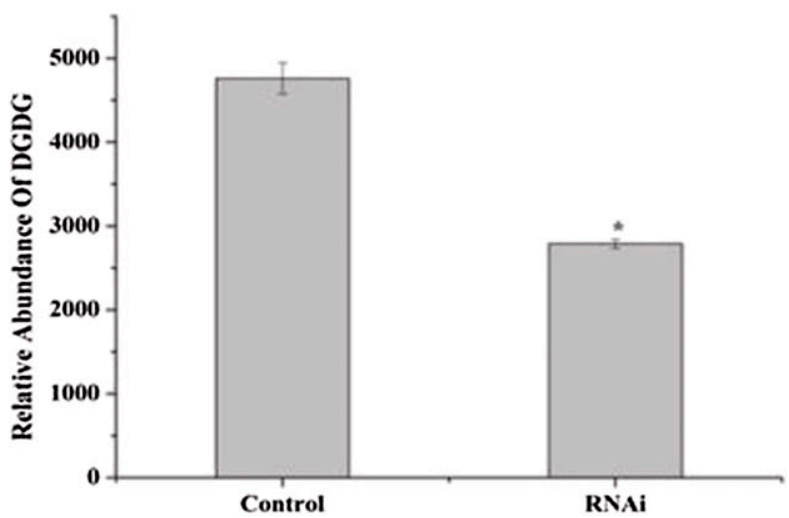

Fig. 6. Nodulation phenotype and digalactosyldiacylglycerol (DGDG) abundance of nodules in MtDGD1-RNA interference (RNAi) transgenic plants. A, Appearances of the control (the left) and MtDGDI-RNAi hairy root plants (the right); $\mathbf{B}$, appearance of root nodules on control hairy roots; $\mathbf{C}$, appearance of root nodules on MtDGD1-RNAi hairy roots; D, relative transcript levels of MtDGD1, MtDGD2, and MtEF1- $\alpha$ in overexpression nodules; E, DGDG content in MtDGD1-RNAi nodules. Asterisks: ** indicates extremely significant differences at the $P<0.01$ level (Dunnett's multiple comparison test) and * indicates significant differences at the $P<0.05$ level. All of the transcript levels were determined using GAPDH as the internal standard gene. Error bars represent the standard deviations of three independent experiments. Bars $=2 \mathrm{~cm}(\mathrm{~A})$ and $2 \mathrm{~mm}(\mathrm{~B}$ and $\mathrm{C}$ ). 
and control nodules was conducted by transmission electron microscopy (TEM). The results showed that the symbiosomes of the control nodules developed normally, with rounded smooth boundaries and homogeneous cytoplasm (Fig. 7E and $\mathrm{G})$; in contrast, most of the symbiosomes in the MtDGD1RNAi nodule cells were smaller in size and displayed abnormal morphologies. In addition, the symbiosomes in MtDGD1-RNAi nodules exhibited enlarged PBM spaces and wrinkled membranes. Most symbiosomes showed severe shrinking and significant increases in electron density, with a quite dark color (Fig. 7F and $\mathrm{H}$ ). These characteristics might be attributed to the accumulation of precursors for DGDG synthesis in the symbiosomes. Overall, it could be inferred that MtDGDI RNAi impaired nodule development and nitrogen fixation. Based on these findings, it can be hypothesized that MtDGD1 plays an essential role in symbiosome development and nitrogen fixation.

\section{Knockdown of MtDGD1 affected the abundance of DGDG in the nodules.}

LC-MS was performed to measure the abundance of DGDG in MtDGD1-RNAi nodules. The results showed that the abundance of DGDG in MtDGD1-RNAi nodules was significantly decreased by approximately $50 \%$ compared with that of the control, suggesting that MtDGDl participates in DGDG synthesis in the nodules of $M$. truncatula (Fig. 6E).

\section{Low phosphorus treatment affected MtDGD1 expression and lipid abundance in the roots and nodules.}

Under phosphorus starvation, the synthesis of phospholipids was decreased while that of galactolipids was increased. This membrane lipid remodeling is a defense mechanism of plants to survive under low phosphorus conditions (Klaus et al. 2002; Shimojima et al. 2015). In this study, we investigated the influence of low phosphorus treatment on the transcription of $M t D G D 1$ and lipid abundance in the roots and nodules under nonsymbiotic or symbiotic conditions.

Under nonsymbiotic conditions, the MtDGDl transcript levels in the uninoculated roots after low-phosphorus (LP) Hoagland's treatment ( $1 \mathrm{mmol} \mathrm{KH}_{2} \mathrm{PO}_{4}$ in Hoagland's was replaced by $5 \mu \mathrm{mol} \mathrm{KH}_{2} \mathrm{PO}_{4}$ ) for 21 days were 13 -fold higher than those in the control watered with normal Hoagland's (Hoagland and Arnon 1950; Xie et al. 2013). At the same time, the transcription of MtDGD2 was also induced by low phosphorus treatment (up to 2.7-fold higher than the control) (Fig. 8A). A phosphate transporter gene MtPT2 (AF000355.1), whose expression was enhanced under low phosphorus conditions in M. truncatula (Liu et al. 1998), was used as a positive control in this study. The results indicated that the transcript level of MtPT2 was significantly increased under low phosphorus conditions (Fig. 8A). The transcript level of $M t E F 1-\alpha$ was quite stable (Fig. 8A). The abundance of all phospholipids such as PC, PE, and PI decreased, while that of nonphospholipid DGDG significantly increased by threefold compared with that in the control (Fig. 8B). In addition, to examine the effect of low phosphorus treatment on MtDGDI expression and lipid level under symbiotic conditions, the plants watered with NFS (Fahraeus 1957) or suffered from LP NFS (LP NFS: $\mathrm{Na}_{2} \mathrm{HPO}_{4}$ in NFS was replaced by $\mathrm{Na}_{2} \mathrm{SO}_{4}$ at 0.4 mmol liter ${ }^{-1}$, and $\mathrm{KH}_{2} \mathrm{PO}_{4}$ at $0.1 \mathrm{~g} \mathrm{liter}^{-1}$ was replaced by $\mathrm{KH}_{2} \mathrm{PO}_{4}$ at $5 \mu \mathrm{mol}$ liter $^{-1}$ and $\mathrm{KCl}$ at $0.7 \mathrm{mmol} \mathrm{liter}^{-1}$ ) (Wang et al. 2015) were inoculated with $S$. meliloti 1021 . The results showed that the transcript levels of MtDGD1 in roots and nodules were elevated to 17- and 2-fold, respectively, of that in the control at $21 \mathrm{dpi}$. However, the transcript level of MtDGD2 displayed no significant changes in both the roots and nodules. The transcript level of the control gene MtPT2 was 2.7- and 6-fold that in the control roots and nodules, respectively, while that of the control gene MtEF1- $\alpha$ showed little change (Fig. 9A). In addition, the content of DGDG in the LP roots and nodules was increased to three- and twofold, respectively, of that in the control watered with normal NFS. However, the contents of PC, PE, and PI were reduced by $94.8,59.4$, and $44.9 \%$ in the LP roots and by $41.2,58.1$, and $36.6 \%$ in the nodules, respectively (Fig. 9B). These data showed that the expression of MtDGD1 and the abundance of DGDG increased correspondingly in the roots and nodules under phosphorus starvation, whereas the phospholipids were reduced.

These results indicated that the membrane lipids in legume host plants are remodeled under nitrogen and phosphorus nutrient deficiency, resulting in the accumulation of DGDG
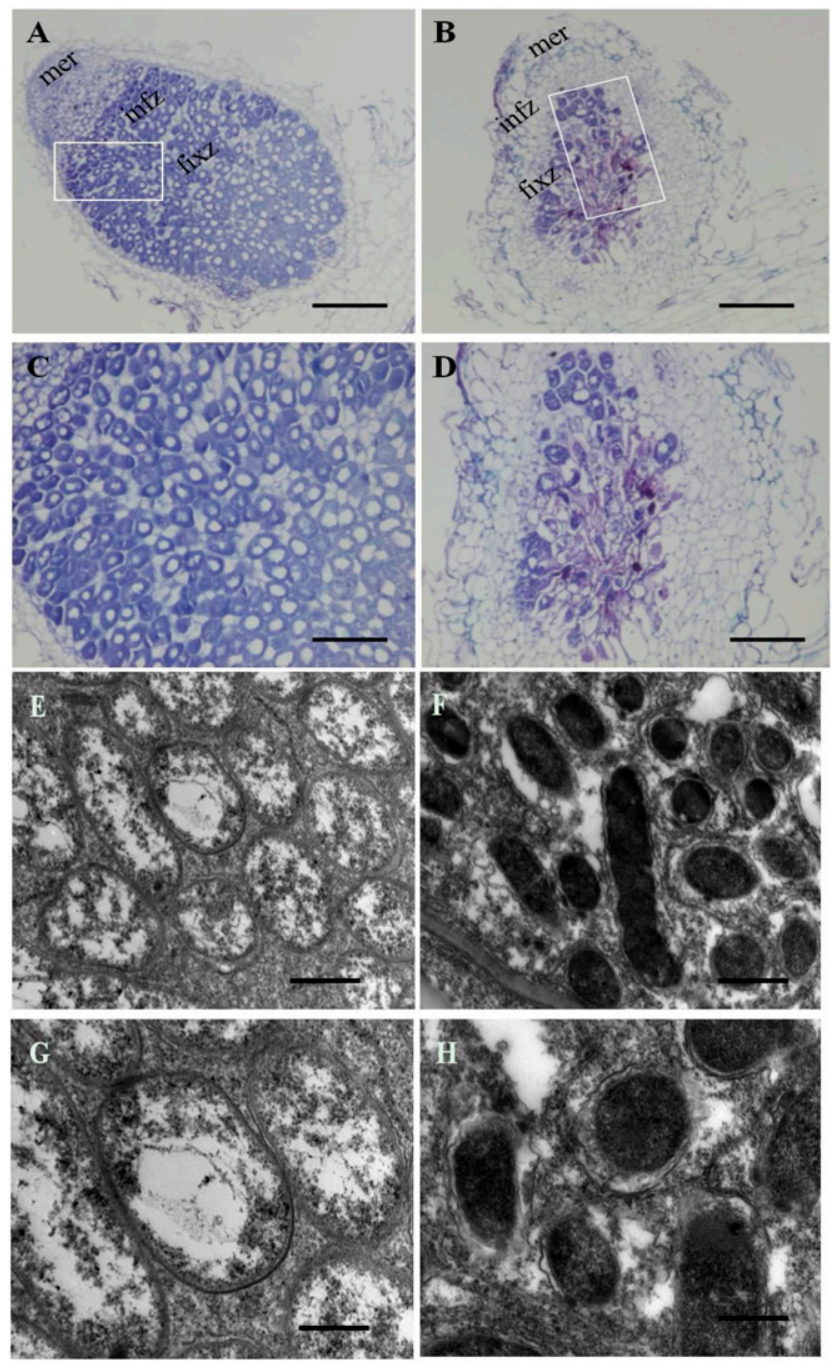

Fig. 7. Microscopic observations of the development of root nodules and symbiosomes in MtDGD1-RNA interference (RNAi) experiment. A to $\mathbf{D}$, Paraffin sections of nodules and toluidine blue staining. A, Longitudinal section of nodule at 28 days postinoculation (dpi) from the control with empty vector; $\mathbf{B}$, longitudinal section of a $M t D G D 1$-RNAi nodule at $28 \mathrm{dpi}$; $\mathbf{C}$, magnification of nitrogen-fixing zone (fixz) in A; and D, Magnification of fixz in B. Abbreviations: mer $=$ meristem and infz $=$ infection zone. Bars $=$ $500 \mu \mathrm{m}$ (A and B) and $100 \mu \mathrm{m}$ (C and D). $\mathbf{E}$ to $\mathbf{H}$, Transmission electron microscopy (TEM) images of symbiosomes of nodule. E, TEM images of symbiosomes of the control nodule with empty vector at $28 \mathrm{dpi}$; F, TEM images of symbiosomes of MtDGD1-RNAi nodule at $28 \mathrm{dpi}$; G, magnification of symbiosomes in $\mathrm{E}$; and $\mathbf{H}$, magnification of symbiosomes in $\mathrm{F}$. Bars $=1 \mu \mathrm{m}(\mathrm{E}$ and $\mathrm{F})$ and $500 \mathrm{~nm}(\mathrm{G}$ and $\mathrm{H})$. 
in the roots and nodules to facilitate symbiotic nitrogen fixation.

\section{DISCUSSION}

Glycerol glycolipids were first extracted and identified in wheat (Carter et al. 1956), and were reported to be the main components of the photosynthetic membranes in cyanobacteria and higher plants (Kobayashi et al. 2007; Murata and Nishida 1987). As the main lipid of the thylakoid membrane, DGDG is also present on the PBMs of the legume plants G. max and L. japonicas. This finding indicates that DGDG plays an essential role in photosynthesis and symbiotic nitrogen fixation (Dörmann et al. 1999; Gaude et al. 2004; Sato and Awai 2016).

MtDGDl was identified as a galactosyltransferase-like protein that could catalyze the transfer of sugar moieties from activated donor molecules to specific acceptor molecules to form glycosidic bonds (Albesa-Jové et al. 2014; Blanco Capurro et al. 2017; Kozmon and Tvaroška 2006). DGDG is a kind of glycoconjugate. It is possible that the biosynthesis of DGDG in M. truncatula is similar to that in Arabidopsis, in which DGDG is produced by transferring a galactosyl residue
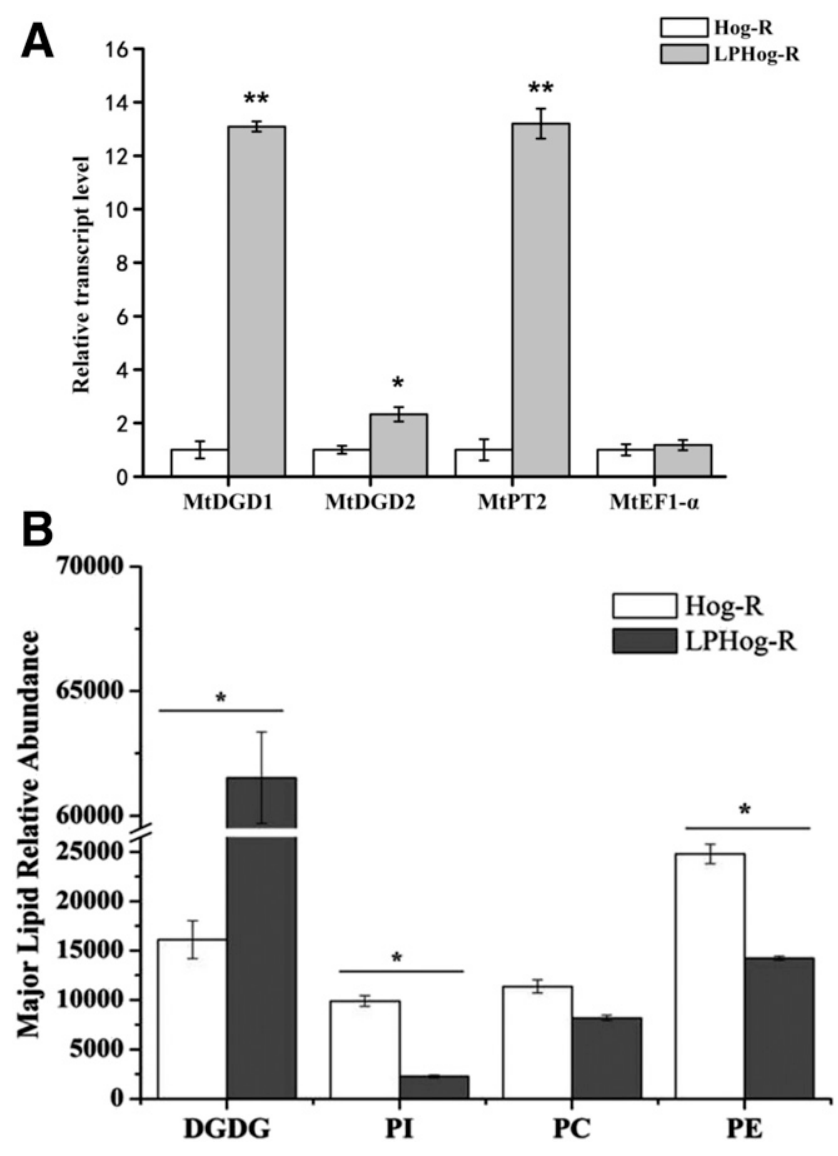

Fig. 8. $M t D G D 1$ expression and digalactosyldiacylglycerol abundance in roots in response to low phosphorus (LP) under nonsymbiotic conditions. A, Transcript levels of $M t D G D 1, M t D G D 2, M t P T 2$, and $M t E F 1-\alpha$ in uninoculated roots after LP treatment on day 21. B, Abundance of different lipid components in uninoculated roots after LP treatment on day 21. Asterisks: * indicates significant differences between the two groups $(P<$ $0.05)$ and $* *$ indicates extremely significant differences between the two groups $(P<0.01)$. All of the transcript levels were determined using $G A P D H$ as the internal standard gene. Error bars represent the standard deviations of three independent experiments. from uridine diphospho-galactose to MGDG (Holzl and Dörmann 2007).

However, the functional phenotype and mechanism of DGDG in nodule development and symbiotic nitrogen fixation still remain elusive. In particular, the genes involved in DGDG synthesis during nodule symbiosis have not been elucidated. In this study, we identified the symbiotic phenotypes of the $M t D G D 1$ gene which encode a DGDG synthase in M. truncatula. Direct experimental evidence was obtained to characterize the function and phenotype of the MtDGDl gene during early infection and nodule symbiosis. The results provide novel insights into DGDG synthesis and its significance in nodule development and nitrogen fixation.

Our results revealed that $M t D G D 1$ plays an important role in the synthesis of DGDG in nodules under symbiotic conditions. The real-time quantitative PCR (qPCR) showed that MtDGD1 expression was induced by rhizobia, and was significantly upregulated in the stems, leaves, and roots. The spatial
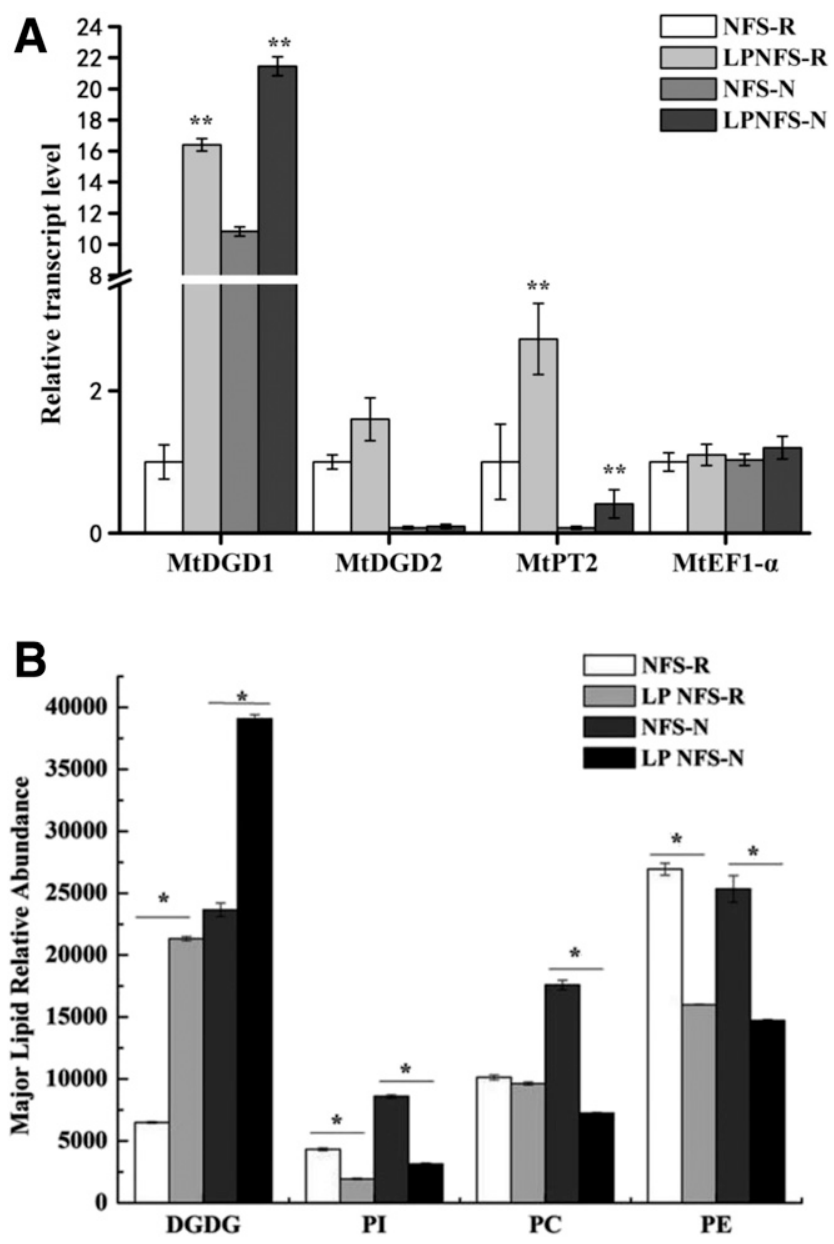

Fig. 9. MtDGD1 expression and digalactosyldiacylglycerol (DGDG) abundance in roots and nodules in response to low phosphorus (LP) under symbiotic conditions. A, Transcript levels of MtDGD1, MtDGD2, MtPT2, and MtEF1- $\alpha$ in infected roots and nodules after LP treatment on day 21 and $\mathbf{B}$, abundance of different lipid components in infected roots and nodules after LP treatment on day 21 . Asterisks: * indicates significant differences between the two groups $(P<0.05)$ and $* *$ indicates extremely significant differences between the two groups $(P<0.01)$. All of the transcript levels were determined using GAPDH as the internal standard gene. NFS = nitrogen-free Fahraeus nutrient solution, LP NFS = nitrogen free solution with low phosphorus, $\mathrm{R}=$ infected roots, $\mathrm{N}=$ nodules, $\mathrm{PI}=$ phosphatidylglycerol, $\mathrm{PC}=$ phosphatidylcholine, and $\mathrm{PE}=$ phosphatidylethanolamine. Error bars represent the standard deviations of three independent experiments. 
expression analysis of $M t D G D 1$ revealed a dynamic expression pattern of MtDGDl during nodule development and, in particular, higher expression of $M t D G D 1$ was observed in the infection zone of young nodules. These observations suggest that the biosynthesis of DGDG is important for the whole progress of nodule development and nitrogen fixation. In addition, immunofluorescence and immunoelectron microscopy experiments also supported the hypothesis that MtDGDl participates in DGDG synthesis. MtDGD1 was observed in the infected cells of nodules and was colocalized with bacteroids, suggesting that MtDGDl is localized on the symbiosome membranes, and plays an important role in symbiosome development and lipid synthesis (Kelly and Dörmann 2002). Meanwhile, there was a significant decrease in DGDG content in the nodules after $M t D G D 1$ silencing. All of these results support the hypothesis that MtDGD1 is a key gene involved in DGDG synthesis during nodule development. The findings provide new insights into the mechanism of membrane lipid biosynthesis and metabolism during legume-rhizobia symbiotic interaction.

Our results indicated that $M t D G D 1$ plays an essential role in nodule development and nitrogen fixation by affecting DGDG synthesis. DGDG is one of the main components of the symbiosome membranes and is essential for maintaining the integrity and stability of the symbiosome membrane structures required for effective symbiotic nitrogen fixation. In contrast to the control, MtDGD1-RNAi plants showed significant decreases in the abundance of DGDG in the nodules, which severely impaired the nodule development and nitrogen fixation, and ultimately resulted in the formation of fewer and smaller nodules. Some of the nodules were found to be white, with much lower nitrogenase activities and abnormal symbiosome morphologies, leading to nitrogen deficiency in the MtDGD1RNAi plants. As a result, the plant growth was hindered. In addition, overexpression of MtDGDl increased the abundance of DGDG in the nodules as well as the number of nodules in transgenic plants but no other differences in symbiotic phenotype were observed between $M t D G D 1$ overexpressing transgenic plants and the control, suggesting that the increase in DGDG would facilitate nodulation. All the findings of this study are consistent with a previous report on the functional phenotypes of a lipid transfer protein gene AsE246 in symbiotic nitrogen fixation (Lei et al. 2014). It should be noted that, in a recent study of the function mechanism of another lipid transfer protein gene, MtN5, in M. truncatula, overexpression of MtN5 changed the lipid profiles of rhizobia-inoculated MtN5ox roots. Several lipids, including some DGDGs, one MGDG, the PE phospholipid, and the two GlcCer, showed higher signals in MtN5ox roots than in the WT roots, and the numbers of invaded primordia and mature nodules per plant were significantly increased in comparison with those in WT plants (Santi et al. 2017). All of these findings further confirm the essential role of DGDG in nodule formation and nitrogen fixation. In summary, our results suggest that MtDGD1 is involved in DGDG synthesis in nodules, and that this synthesis is essential for the development of symbiosomes and nitrogen fixation.

Taken together, our results demonstrate that DGDG has important biological functions in the progression of symbiosis and, thus, the possible functional mechanism should be further dissected.

First, the accumulation of the nonphospholipid DGDG under phosphorus starvation indicates that DGDG plays an important role in membrane lipid remodeling under the conditions of nitrogen and phosphorus nutrient deficiency. Because phosphorus is essential to plant growth and development, the effective supply of phosphorus can significantly promote the development of nodules and nitrogen fixation of plants, especially under nitrogen-deficient conditions (Leidi and
Rodríguez-Navarro 2000). Under phosphorus and nitrogen deficiency, DGDG instead of phospholipids can accumulate on the symbiosome membranes for possible membrane remodeling. This process could preserve the phosphorus elements for other important cellular activities in nodule development and nitrogen fixation. For normal and successful nodule development, phosphorus saving is necessary to meet the demand of the synthesis of phosphorus-containing biological macromolecules such as phosphorus-related proteins and nucleic acids during nodule development and nitrogen fixation (Narasimhan et al. 2013). In response to phosphorus stress, the components of the symbiosome membranes will be changed, and the original phospholipids will be degraded or replaced by nonphospholipid DGDG, which is a common phenomenon in plants. In brief, the accumulation of DGDG under symbiosis may also be a unique strategy of leguminous plants to preserve phosphorus for nodule development, particularly under phosphorous starvation.

Second, a previous study has reported a significant correlation between the MGDG and DGDG content in wheat leaves and resistance to wheat leaf spot caused by pathogenic fungi (Kim et al. 2013). Moreover, (Santi et al. 2017) reported that several genes involved in the response to biotic stress were modulated in MtN5hp roots, and two proteins (Medtr4g021037 and Medtr8g446950) involved in disease resistance and a cationic peroxidase (Medtr7g107520) were upregulated, suggesting that the content of glycolipids can affect disease resistance. In this study, a dynamic expression pattern was observed for MtDGDI in the infection zone of young nodules and in the entire mature nodules, suggesting the importance of DGDG biosynthesis in nodule development and symbiotic nitrogen fixation. Considering that root nodule formation is the outcome of interaction and coordination between symbiosis and defense, DGDG biosynthesis might be a possible strategy for the intracellular symbiosis between plants and rhizobia.

Finally, in the $d g d l$ mutant of Arabidopsis, some excess MDGDs were converted into a large quantity of jasmonic acid (JA), causing visible phenotypes such as extremely short inflorescence stems, lignified phloem cap cells, and ruffled leaves (Lin et al. 2016). This finding suggests that DGDl might also indirectly affect the symbiotic phenotypes by increasing JA levels, because JA was reported to be associated with nodule formation and nitrogen fixation (Isayenkov et al. 2005; Kinkema and Gresshoff 2008).

\section{MATERIALS AND METHODS}

\section{Plant materials.}

The accession Jemalong A17 of M. truncatula was used in this study. Seed were scratched lightly with a needle, surface sterilized in 2\% hypochlorite (commercial bleach) for 2 to $5 \mathrm{~min}$, then washed several times in sterile water. The seed were then immersed in distilled water for $3 \mathrm{~h}$ and placed in a medium with $0.5 \%$ sucrose and $1.2 \%$ agar for germination (Garcia et al. 2006). Germinated seed were planted in pots filled with sterile sand and watered with NFS. After cotyledon expansion, the plantlets were inoculated with or without $S$. meliloti 1021. All plants were grown in a greenhouse under daily conditions of $16 \mathrm{~h}$ of light and $8 \mathrm{~h}$ of darkness at 24 and $20^{\circ} \mathrm{C}$, respectively.

\section{Bioinformatic and phylogenetic analyses.}

Based on the query sequences AY635907 (Gm-DGD1), AY635908 (Gm-DGD2), Y635909 (Lj-DGD1), and AY635910 ( $L j-D G D 2)$ (Gaude et al. 2004), two DGDG synthase 1 and four DGDG synthase 2 were obtained from the M. truncatula genome by blasting on the NCBI Standard Nucleotide BLAST website. The transcript levels of different DGDG synthases in 
different tissues of $M$. truncatula were obtained from the transcriptome data (Young et al. 2011). The physical and chemical parameters of MtDGD1 were predicted using the ExPASy website. The sequences of MtDGD1 and MtDGD2 were blasted on the BLAST website, and their similarities to the homologs in higher plants, including other leguminous plants, were detected. Conserved domain analysis was performed on the NCBI conserved domains website. Amino acid sequence alignment was performed by DNAMAN software.

MtDGD1 homologous proteins were downloaded from the genomes of M. truncatula (Mt), L. japonicus, G. max, Phaseolus vulgaris, Arabidopsis thaliana, Oryza sativa, and $\mathrm{Ni}$ cotiana benthamiana from phytozome v11.0 and the NCBI database. All alignments were performed by using ClustalW method. The maximum-likelihood method was used for the phylogenetic analysis. The phylogenetic tree was constructed with MEGA6.0 software. Bootstrap values were from 1,000 replications

\section{Cloning of full-length MtDGD1 cDNA and plasmid construction.}

Nodules were harvested at 40 dpi. Total RNA was extracted with the TRIzol reagent (Invitrogen) according to the manufacturer's instructions. The primers of full-length $M t D G D 1$ cDNA were 5' GAGCTCATGGCTACACAGCATCCACCTTC $3^{\prime}$ and 5' CTGCAGCACAGTACAACTACAACTGAAC 3'. To construct the overexpression plasmid, the open reading frame of MtDGDl was amplified by reverse-transcription PCR using the abovementioned primers containing a SacI site and a SalI site at each $5^{\prime}$ end of the primer. The amplification product was digested with SacI-SalI and ligated into the pCAMBIA 2301 vector (Gene ID: AF234316). To generate the RNAi, a 287-bp fragment with a short part of the coding region of MtDGDl in the $3^{\prime}$ untranslated region was amplified by reversetranscription PCR using the following primers: 5' GAGCTC CACTATTGTCTCACAGGGAACG $3^{\prime}$ containing a BamHI or Pst site at the 5' end and 5' GGATCCAACAAGCAGAAA GCCGAACTAC $3^{\prime}$ containing a $S a c I$ or $X b a I$ site at the $5^{\prime}$ end. The amplification products were digested with BamHI-SacI or with Pstl-Xbal and ligated into the pCAMBIA1301-35S-int-T7 plasmid vector (Chen et al. 2012). To generate the promoterGUS vector, the promoter of $M t D G D 1$ was amplified with the following primers: 5' GCAGGTCGACGGATCCCCGGGGTT AAACCTATTAACTTGTCAAATG 3 'containing 15 bp of pDX2181G-mCherry vector at a SmaI site upstream and 5' ATAAGGGACTGACCACCCGGGGCTTTTGTCCTATATATCA ACAATC 3 ' containing 15 bp of pDX2181G-mCherry vector at a SmaI site downstream at each $5^{\prime}$ end. The amplification product and pDX2181G-mCherry vector, a pDX2181derivative (Ye et al. 2012), were linked with recombinase. The constructs were transferred into Agrobacterium rhizogenes MSU 440 by electroporation.

\section{Antibody preparation.}

The MtDGD1 coding sequence (accession number XM_ 013594759) was amplified from complementary DNA. The upstream primer was 5' CATATGGCTACACAGCATCCACCTT 3' and the downstream primer was 5' GCGGCCGCACCAGCCA TAGATAGGACTTTC $3{ }^{\prime}$. Considering that MtDGD1 and MtDGD2 had a high homology in the C-terminal sequence, the coding sequence of MtDGD1 was double digested into an $\mathrm{N}$ terminal truncated fragment (576 bp, encoding 189 aa) with $N d e I$ and HindIII. This truncated fragment was distinguished from MtDGD2 and then cloned into pET-28a(+). The fragment sequence was confirmed by sequencing. The recombinant vector pET-28a-MtDGD1 was transformed into the host strain Escherichia coli BL21 DE3. The MtDGD1-truncated protein was purified from the E. coli lysate utilizing an HIS cartridge (Bio-Rad).
Purified MtDGD1 protein was used for immunization in New Zealand white rabbits to obtain the anti-MtDGD1 antibody.

\section{RNA extraction and real-time PCR.}

To study the expression patterns of MtDGDl during nodule development, leaves, stems, roots, and nodules were harvested at 21 dpi with or without $S$. meliloti 1021 inoculation. The leaves and nodules inoculated with $S$. meliloti 1021 were harvested at different times (days 5, 9, 15, 21 and 32). The LP stress experiment was performed as described previously (Wang et al. 2015). When the first cotyledon was fully extended, under nonsymbiotic conditions, the seedling was treated with Hoagland's or LP Hoagland's, with $1 \mathrm{mmol}$ $\mathrm{KH}_{2} \mathrm{PO}_{4}$ in Hoagland's being replaced by $5 \mu \mathrm{mol} \mathrm{KH}_{2} \mathrm{PO}_{4}$; while, under the symbiotic conditions, the seedling was treated with NFS (Fahraeus 1957) or LP NFS, with $\mathrm{Na}_{2} \mathrm{HPO}_{4}$ in NFS being replaced by $0.4 \mathrm{mmol} \mathrm{Na} \mathrm{SO}_{4}$ and $\mathrm{KH}_{2} \mathrm{PO}_{4}$ at $0.1 \mathrm{~g}$ liter $^{-1}$ being replaced by $5 \mu \mathrm{mol} \mathrm{KH}_{2} \mathrm{PO}_{4}$ and $0.7 \mathrm{mmol} \mathrm{KCl}$. The roots or nodules of $M$. truncatula were harvested on day 21 .

Total RNA was extracted from each tissue sample using Trizol reagent (Invitrogen) according to the manufacturer's instructions. Genomic DNA was eliminated by DNAaseI (Fermentas). The purity and concentration of each RNA sample were measured by a Nano Drop 2000 spectrophotometer (Thermo Scientific). After adjusting the amount of RNA, the cDNA was synthesized by RevertAid rReverse transcription (Fermentas) using oligo(dT)18 primers. The fluorescence qPCR was performed using the Faststart Universal SYBR Green Master Kits (Roche), and qPCRs were monitored and analyzed using the Applied Biosystems (ABI) StepOne. The transcript levels of MtDGD1, MtDGD2, $M t P T 2, M t G A P D H, M t E F 1-\alpha$, and MtCOPT1 were assayed by fluorescence qPCR. MtGAPDH served as an internal standard gene, and housekeeping gene MtEFl- $\alpha$ served as the other reference gene. All of the real-time PCR primers were listed in the Supplementary Table S2. The data were analyzed using the $2^{-\Delta \Delta \mathrm{CT}}$ method. Each experiment was repeated at least three times.

\section{Immunofluorescence experiment.}

Nodules were hand sectioned using a double-edged razor blade. Nodule sections or roots were fixed in $1 \%(\mathrm{wt} / \mathrm{vol})$ freshly depolymerized paraformaldehyde in phosphatebuffered saline (PBS) at $\mathrm{pH} 7.4$ for $30 \mathrm{~min}$ at $4^{\circ} \mathrm{C}$. Nodule sections were blocked in $3 \%(\mathrm{wt} / \mathrm{vol})$ bovine serum albumin (BSA) and further incubated with the primary antibody overnight at $4{ }^{\circ} \mathrm{C}$ in PBS containing $0.3 \%$ (vol/vol) Triton X-100. The secondary antibody (goat antirabbit) conjugated with CY3, a red fluorescence dye, was used. The control experiment was performed without adding primary antibodies or with the addition of the preimmune sera. Nodule sections containing GFP-labeled S. meliloti 1021 were examined by a confocal microscopy. Antibody dilution was prepared as follows: antiMtDGD1 antibody at 1:100 and goat antirabbit (CY3) at 1:1,000.

\section{Microscopic analysis.}

Microscopic analysis was performed as previously described (Li et al. 2008). The 28-dpi nodules were analyzed. For light microscopy, nodules were cut longitudinally, fixed in formalin-aceto-alcohol buffer, washed, dehydrated, and embedded in paraffin, and the slides were stained with toluidine blue, then observed with an Olympus light microscope. For electron microscopy analysis, nodules were fixed in $2.5 \%$ glutaraldehyde for $4 \mathrm{~h}$, then post fixed in $1 \%$ osmium tetroxide for $3 \mathrm{~h}$, washed, dehydrated through an ethanol series, and embedded in London resin white. Ultrathin sections were examined by TEM (H-7650; Hitachi). 


\section{Immunoelectron microscopy analysis.}

Immunoelectron microscopy analysis was performed as reported previously (Limpens et al. 2009). The nodules were fixed by $4 \%$ paraformaldehyde in $2.5 \%$ glutaraldehyde, and postfixed with $1 \%$ osmium terroxide for $3 \mathrm{~h}$. After washing and dehydrating through an ethanol series, the tissues were embedded in LR White and polymerized at $60^{\circ} \mathrm{C}$. Then, the nodules were cut into ultrathin sections $(60 \mathrm{~nm})$ by a Leica EM UC7 ultramicrotome. Nickel grids with sections $(60 \mathrm{~nm})$ were blocked with $2 \%$ (wt/vol) BSA in PBS and incubated with the primary antibody overnight at $4^{\circ} \mathrm{C}$ in PBS according to the dilutions described above. Goat antirabbit antibody coupled with $10 \mathrm{~nm}$ gold (1:1,000 dilution) was used as the secondary antibody and incubated for $2 \mathrm{~h}$. The sections were examined by TEM (H-7650; Hitachi).

\section{Plant transformation.}

A. rhizogenes MSU440 cells containing the binary vectors were used to induce hairy root formation on M. truncatula seedlings, as described previously (Chabaud et al. 2006; Limpens et al. 2004). Briefly, radicles approximately $3 \mathrm{~mm}$ in length were cut from the root tip with a sterile scalpel when the seedlings had a radicle length of approximately $1 \mathrm{~cm}$. Then, the sectioned surface was coated with $A$. rhizogenes by light scraping. The seedlings were transferred onto agar with modified Fahraeus medium and cocultivated with A. rhizogenes MSU440 for 5 days in a growth chamber. The plants were transferred onto hairy root emergence medium plates containing timentin at $300 \mu \mathrm{g} / \mathrm{ml}$ and grown for 10 more days until the development of hairy roots from the hypocotyls. To select the transgenic hairy roots, root tips of 2 to $3 \mathrm{~mm}$ were excised for GUS staining overnight at $37^{\circ} \mathrm{C}$ in a solution containing $100 \mathrm{mM}$ sodium phosphate ( $\mathrm{pH} 7.0$ ), $0.1 \%$ Triton $\mathrm{X}-100,0.1 \%$ N-lauroylsarcosine, $10 \mathrm{mM}$ EDTA, $1 \mathrm{mM} \mathrm{K} \mathrm{Fe}_{3}(\mathrm{CN})_{6}, 1 \mathrm{mM}$ $\mathrm{K}_{4} \mathrm{Fe}(\mathrm{CN})_{6}$, and 5-bromo-4-chloro-3-indoxyl- $\beta$-D-glucuronide cyclohexylammonium salt at $0.5 \mathrm{mg} / \mathrm{ml}$. The remaining portion of the hairy root was labeled. If the hairy root tip was GUS negative, the whole hairy root would be discarded; if it was GUS positive, the remaining portion of the hairy root would be retained. One to three transgenic hairy roots were preserved for each seedling. Plants harboring transgenic hairy roots were transferred to pots filled with vermiculite and sand (1:1) and grown in a chamber with a cycle of $16 \mathrm{~h}$ (day) and $8 \mathrm{~h}$ (night)e at 24 and $20^{\circ} \mathrm{C}$, respectively. After 5 to 7 days, the plants were inoculated with $S$. meliloti 1021. Symbiotic phenotypes were examined 4 weeks after inoculation.

\section{Nitrogenase activity measurement.}

Nitrogenase activity was measured with the method of the acetylene reduction activity (Wych and Rains 1978). Nine hairy root lines from each sample were analyzed. The hypogeal parts, including the nodules and roots, were incubated for $2 \mathrm{~h}$ at $28^{\circ} \mathrm{C}$ in 30-ml glass bottles containing $2 \mathrm{ml}$ of $\mathrm{C}_{2} \mathrm{H}_{2}$. The amount of ethylene was measured using an East \& West Analytical Instrument GC 4000A gas chromatograph.

\section{Lipid extraction and quantification.}

To determine the lipid composition of the roots and nodules, plant tissues at different stages were harvested $(100 \mathrm{mg})$ for lipid extraction, and were homogenized in liquid nitrogen (Bligh and Dyer 1959). Samples were resuspended in $50 \mathrm{ml}$ of chloroform and $100 \mathrm{ml}$ of methanol. Subsequently, they were sonicated three to five times. Afterward, $50 \mathrm{ml}$ of chloroform and $50 \mathrm{ml}$ of distilled and deionized water were added to the mixture. Then, the solution was transferred to a separatory funnel and allowed to stand for approximately $30 \mathrm{~min}$ to achieve complete clarification and separation. Finally, lipids extracted from the lower organic phase were dried by rotary evaporation, then dissolved in methanol (Lei et al. 2014).

The lipid extracts were introduced into a quadrupole time-offlight (Q-TOF) mass spectrometer (Agilent 6540 Accurate Mass Q-TOF LC-MS unit) operating in the positive mode. Lipids were supplied to the mass spectrometer in methanol. Q-TOF LC-MS was performed in an Agilent 6540 UHD accurate-mass Q-TOF LC-MS system with a Zorbax Eclipse Plus C18 column $(2.1 \times$ $100 \mathrm{~mm}, 3.5 \mu \mathrm{m})$. The sample of $1 \mu \mathrm{l}$ was loaded at a flow rate of $0.3 \mathrm{ml} \mathrm{min}^{-1}$ in solvent A (5\% methanol and $\left.10 \mathrm{mM} \mathrm{NH}_{4} \mathrm{AcOA}\right)$ and solvent B (methanol). Solvent B was $25 \%(\mathrm{vol} / \mathrm{vol})$ at the start, $100 \%(\mathrm{vol} / \mathrm{vol})$ at $5 \mathrm{~min}, 100 \%(\mathrm{vol} / \mathrm{vol})$ at $10 \mathrm{~min}$, and $25 \%$ ( $\mathrm{vol} / \mathrm{vol}$ ) again at $15 \mathrm{~min}$. The acquisition range was 400 to 1,100 $\mathrm{m} / \mathrm{z}$; nebulizer pressure, 40 pounds per square inch gauge; drying gas, $\mathrm{N}_{2}$ at $350^{\circ} \mathrm{C}$ and 9 liters $\mathrm{min}^{-1}$; electrospray ionization voltage of capillary, 4,000 V; fragment of $214 \mathrm{v}$; skimmer, $65 \mathrm{~V}$; and octopole radio frequency programming mode voltage, $750 \mathrm{~V}$. MGDG and DGDG were analyzed as $\left[\mathrm{M}+\mathrm{NH}_{4}\right]^{+}$and $[\mathrm{M}+\mathrm{Na}]^{+}$ ions, respectively (Wu and Xue 2010).

\section{Statistical analysis.}

Statistical significance was analyzed using independentsample $t$ test and single-factor analysis of variance method by IBM SPSS Statistics, version 20. Three biological replicates were carried out for each experiment. The bars represent the standard deviations of three independent experiments in the figure legends. $P$ values $<0.05$ or $<0.01$ (Dunnett's multiple comparison test) were considered statistically significant.

\section{ACKNOWLEDGMENTS}

We thank Z. Zhang for providing pCAMBIA1301-35S-int-T7 for the RNAi experiment and pDX2181G-mCherry vector for the GUS staining experiment, and Z. Liu from the Foreign Language School of HZAU for checking and polishing the English language of the manuscript.

\section{AUTHOR-RECOMMENDED INTERNET RESOURCES}

Phytozome v11.0: https://phytozome.jgi.doe.gov/pz/portal.html\#!search?show=BLAST \&method=Org_Mtruncatula

Gene expression profiles in Medicago truncatula: https://iant.toulouse.inra.fr/symbimics/

NCBI Standard Nucleotide BLAST website: https://blast.ncbi.nlm.nih.gov/Blast.cgi?PROGRAM=blastn\&PAGE_TYPE= BlastSearch\&LINK_LOC=blasthome

ExPASy website: https://web.expasy.org/protparam/

NCBI BLAST website: https://blast.ncbi.nlm.nih.gov/Blast.cgi

NCBI conserved domains website:

https://www.ncbi.nlm.nih.gov/Structure/cdd/wrpsb.cgi?

RID=U1GG5DSH015\&mode=all

DNAMAN software:

https://dnaman-32-en-001.software.informer.com/

\section{LITERATURE CITED}

Albesa-Jové, D., Giganti, D., Jackson, M., Alzari, P. M., and Guerin, M. E. 2014. Structure-function relationships of membrane-associated GT-B glycosyltransferases. Glycobiology 24:108-124.

Benning, C., and Ohta, H. 2005. Three enzyme systems for galactoglycerolipid biosynthesis are coordinately regulated in plants. J. Biol. Chem. 280:2397-2400.

Blanco Capurro, J. I., Hopkins, C. W., Pierdominici Sottile, G., González Lebrero, M. C., Roitberg, A. E., and Marti, M. A. 2017. Theoretical insights into the reaction and inhibition mechanism of metalindependent retaining glycosyltransferase responsible for mycothiol biosynthesis. J. Phys. Chem. B 121:471-478.

Bligh, E. G., and Dyer, W. J. 1959. A rapid method of total lipid extraction and purification. Can. J. Biochem. Physiol. 37:911-917.

Brewin, N. J. 1990. The role of the plant plasma membrane in symbiosis. Pages 351-375 in: The Plant Plasma Membrane. C. Larsson and I. Møller, eds. Springer, Berlin, Heidelberg, Germany. 
Carter, H. E., McCluer, R. H., and Slifer, E. D. 1956. Lipids of wheat flour. I. Characterization of galactosylglycerol components. J. Am. Chem. Soc. 78:3735-3738.

Chabaud, M., Boisson-Dernier, A., Zhang, J., Taylor, C. G., Yu, O., and Barker, D. G. 2006. Agrobacterium rhizogenes-mediated root transformation. Medicago truncatula handbook. https://www.noble.org/ globalassets/docs/medicago-handbook/agrobacterium-rhizogenes.pdf

Chen, T., Zhu, H., Ke, D., Cai, K., Wang, C., Gou, H., Hong, Z., and Zhang, Z. 2012. A MAP kinase kinase interacts with SymRK and regulates nodule organogenesis in Lotus japonicus. Plant Cell 24: 823-838.

Dörmann, P., Balbo, I., and Benning, C. 1999. Arabidopsis galactolipid biosynthesis and lipid trafficking mediated by DGD1. Science 284: 2181-2184.

Dörmann, P., and Benning, C. 2002. Galactolipids rule in seed plants. Trends Plant Sci. 7:112-118.

Essemine, J., Govindachary, S., Ammar, S., Bouzid, S., and Carpentier, R. 2011. Functional aspects of the photosynthetic light reactions in heat stressed Arabidopsis deficient in digalactosyl-diacylglycerol. J. Plant Physiol. 168:1526-1533.

Fahraeus, G. 1957. The infection of clover root hairs by nodule bacteria studied by a simple glass slide technique. J. Gen. Microbiol. 16:374-381.

Gao, Q. M., Yu, K., Xia, Y., Shine, M. B., Wang, C., Navarre, D., Kachroo, A., and Kachroo, P. 2014. Mono- and digalactosyldiacylglycerol lipids function nonredundantly to regulate systemic acquired resistance in plants. Cell Rep. 9:1681-1691.

Garcia, J., Barker, D. G., and Journet, E. P. 2006. Seed storage and germination. Medicago truncatula handbook. https://www.noble.org/ globalassets/docs/medicago-handbook/seed-storage-germination.pdf

Gaude, N., Tippmann, H., Flemetakis, E., Katinakis, P., Udvardi, M., and Dörmann, P. 2004. The galactolipid digalactosyldiacylglycerol accumulates in the peribacteroid membrane of nitrogen-fixing nodules of soybean and Lotus. J. Biol. Chem. 279:34624-34630.

Härtel, H., Lokstein, H., Dörmann, P., Grimm, B., and Benning, C. 1997. Changes in the composition of the photosynthetic apparatus in the galactolipid-deficient dgd1 mutant of Arabidopsis thaliana. Plant Physiol. 115:1175-1184.

Hoagland, D. R., and Arnon, D. I. 1950. The water-culture method for growing plants without soil. California Agricultural Experiment Station, College of Agriculture, University of California, Berkeley, CA, U.S.A.

Hölzl, G., and Dörmann, P. 2007. Structure and function of glycoglycerolipids in plants and bacteria. Prog. Lipid Res. 46:225-243.

Isayenkov, S., Mrosk, C., Stenzel, I., Strack, D., and Hause, B. 2005. Suppression of allene oxide cyclase in hairy roots of Medicago truncatula reduces jasmonate levels and the degree of mycorrhization with Glomus intraradices. Plant Physiol. 139:1401-1410.

Jones, K. M., Kobayashi, H., Davies, B. W., Taga, M. E., and Walker, G. C. 2007. How rhizobial symbionts invade plants: The SinorhizobiumMedicago model. Nat. Rev. Microbiol 5:619-633.

Kelly, A. A., and Dörmann, P. 2002. DGD2, an Arabidopsis gene encoding a UDP-galactose-dependent digalactosyldiacylglycerol synthase is expressed during growth under phosphate-limiting conditions. J. Biol. Chem. 277:1166-1173.

Kelly, A. A., Froehlich, J. E., and Dörmann, P. 2003. Disruption of the two digalactosyldiacylglycerol synthase genes DGD1 and DGD2 in Arabidopsis reveals the existence of an additional enzyme of galactolipid synthesis. Plant Cell 15:2694-2706.

Kim, D., Jeannotte, R., Welti, R., and Bockus, W. W. 2013. Lipid profiles in wheat cultivars resistant and susceptible to tan spot and the effect of disease on the profiles. Phytopathology 103:74-80.

Kinkema, M., and Gresshoff, P. M. 2008. Investigation of downstream signals of the soybean autoregulation of nodulation receptor kinase GmNARK. Mol. Plant-Microbe Interact. 21:1337-1348.

Klaus, D., Härtel, H., Fitzpatrick, L. M., Froehlich, J. E., Hubert, J., Benning, C., and Dörmann, P. 2002. Digalactosyldiacylglycerol synthesis in chloroplasts of the Arabidopsis dgd1 mutant. Plant Physiol. 128:885-895.

Kobayashi, K., Kondo, M., Fukuda, H., Nishimura, M., and Ohta, H. 2007. Galactolipid synthesis in chloroplast inner envelope is essential for proper thylakoid biogenesis, photosynthesis, and embryogenesis. Proc. Natl. Acad. Sci. U.S.A. 104:17216-17221.

Kobayashi, K., Nakamura, Y., and Ohta, H. 2009. Type A and type B monogalactosyldiacylglycerol synthases are spatially and functionally separated in the plastids of higher plants. Plant Physiol. Biochem. 47: 518-525.

Kozmon, S., and Tvaroška, I. 2006. Catalytic mechanism of glycosyltransferases: Hybrid quantum mechanical/molecular mechanical study of the inverting $N$-acetylglucosaminyltransferase I. J. Am. Chem. Soc. 128:16921-16927.

Krumova, S. B., Laptenok, S. P., Kovács, L., Tóth, T., van Hoek, A., Garab, G., and van Amerongen, H. 2010. Digalactosyl-diacylglyceroldeficiency lowers the thermal stability of thylakoid membranes. Photosynth. Res. 105:229-242.

Lastovetsky, O. A., Gaspar, M. L., Mondo, S. J., LaButti, K. M., Sandor, L., Grigoriev, I. V., Henry, S. A., and Pawlowska, T. E. 2016. Lipid metabolic changes in an early divergent fungus govern the establishment of a mutualistic symbiosis with endobacteria. Proc. Natl. Acad. Sci. U.S.A. 113:15102-15107.

Lei, L., Chen, L., Shi, X., Li, Y., Wang, J., Chen, D., Xie, F., and Li, Y. 2014 A nodule-specific lipid transfer protein AsE246 participates in transport of plant-synthesized lipids to symbiosome membrane and is essential for nodule organogenesis in Chinese milk vetch. Plant Physiol. 164:10451058

Leidi, E. O., and Rodríguez-Navarro, D. N. 2000. Nitrogen and phosphorus availability limit $\mathrm{N}_{2}$ fixation in bean. New Phytol. 147:337-346.

Li, Y., Zhou, L., Li, Y., Chen, D., Tan, X., Lei, L., and Zhou, J. 2008. A nodule-specific plant cysteine proteinase, AsNODF32, is involved in nodule senescence and nitrogen fixation activity of the green manure legume Astragalus sinicus. New Phytol. 180:185-192.

Limpens, E., Ivanov, S., van Esse, W., Voets, G., Fedorova, E., and Bisseling, T. 2009. Medicago N2-fixing symbiosomes acquire the endocytic identity marker Rab7 but delay the acquisition of vacuolar identity. Plant Cell 21:2811-2828.

Limpens, E., Ramos, J., Franken, C., Raz, V., Compaan, B., Franssen, H., Bisseling, T., and Geurts, R. 2004. RNA interference in Agrobacterium rhizogenes-transformed roots of Arabidopsis and Medicago truncatula. J. Exp. Bot. 55:983-992.

Lin, Y. T., Chen, L. J., Herrfurth, C., Feussner, I., and Li, H. M. 2016. Reduced biosynthesis of digalactosyldiacylglycerol, a major chloroplast membrane lipid, leads to oxylipin overproduction and phloem cap lignification in Arabidopsis. Plant Cell 28:219-232.

Liu, H., Trieu, A. T., Blaylock, L. A., and Harrison, M. J. 1998. Cloning and characterization of two phosphate transporters from Medicago truncatula roots: Regulation in response to phosphate and to colonization by arbuscular mycorrhizal (AM) fungi. Mol. Plant-Microbe Interact. 11: 14-22.

Moellering, E. R., Muthan, B., and Benning, C. 2010. Freezing tolerance in plants requires lipid remodeling at the outer chloroplast membrane. Science 330:226-228.

Murata, N., and Nishida, I. 1987. Lipids of blue-green algae (cyanobacteria). Pages 315-347 in: Lipids: Structure and Function. Biochemistry of Plants, Vol. 9. Academic Press, London.

Narasimhan, R., Wang, G., Li, M., Roth, M., Welti, R., and Wang, X. 2013. Differential changes in galactolipid and phospholipid species in soybean leaves and roots under nitrogen deficiency and after nodulation. Phytochemistry 96:81-91.

Oldroyd, G. E., Murray, J. D., Poole, P. S., and Downie, J. A. 2011. The rules of engagement in the legume-rhizobial symbiosis. Annu. Rev. Genet. 45:119-144.

Reifarth, F., Christen, G., Seeliger, A. G., Dörmann, P., Benning, C., and Renger, G. 1997. Modification of the water oxidizing complex in leaves of the dgd1 mutant of Arabidopsis thaliana deficient in the galactolipid digalactosyldiacylglycerol. Biochemistry 36:11769-11776.

Santi, C., Molesini, B., Guzzo, F., Pii, Y., Vitulo, N., and Pandolfini, T. 2017. Genome-wide transcriptional changes and lipid profile modifications induced by Medicago truncatula N5 overexpression at an early stage of the symbiotic interaction with Sinorhizobium meliloti. Genes (Basel) 8:396.

Sato, N., and Awai, K. 2016. Diversity in biosynthetic pathways of galactolipids in the light of endosymbiotic origin of chloroplasts. Front. Plant Sci. 7:117

Senovilla, M., Castro-Rodríguez, R., Abreu, I., Escudero, V., Kryvoruchko, I., Udvardi, M. K., Imperial, J., and González-Guerrero, M. 2018 Medicago truncatula copper transporter 1 (MtCOPT1) delivers copper for symbiotic nitrogen fixation. New Phytol. 218:696-709.

Sessions, A., Weigel, D., and Yanofsky, M. F. 1999. The Arabidopsis thaliana MERISTEM LAYER 1 promoter specifies epidermal expression in meristems and young primordia. Plant J. 20:259-263.

Shimojima, M., Madoka, Y., Fujiwara, R., Murakawa, M., Yoshitake, Y., Ikeda, K., Koizumi, R., Endo, K., Ozaki, K., and Ohta, H. 2015. An engineered lipid remodeling system using a galactolipid synthase promoter during phosphate starvation enhances oil accumulation in plants. Front. Plant Sci. 6:664.

Shimojima, M., and Ohta, H. 2011. Critical regulation of galactolipid synthesis controls membrane differentiation and remodeling in distinct 
plant organs and following environmental changes. Prog. Lipid Res. 50: 258-266.

Slack, C. R., Roughan, P. G., and Balasingham, N. 1977. Labelling studies in vivo on the metabolism of the acyl and glycerol moieties of the glycerolipids in the developing maize leaf. Biochem. J. 162:289-296.

Suzaki, T., and Kawaguchi, M. 2014. Root nodulation: A developmental program involving cell fate conversion triggered by symbiotic bacterial infection. Curr. Opin. Plant Biol. 21:16-22.

Wang, J., Si, Z., Li, F., Xiong, X., Lei, L., Xie, F., Chen, D., Li, Y., and Li, Y. 2015. A purple acid phosphatase plays a role in nodule formation and nitrogen fixation in Astragalus sinicus. Plant Mol. Biol. 88:515-529.

Wu, G. Z., and Xue, H. W. 2010. Arabidopsis $\beta$-ketoacyl-[acyl carrier protein] synthase I is crucial for fatty acid synthesis and plays a role in chloroplast division and embryo development. Plant Cell 22: 3726-3744.

Wych, R. D., and Rains, D. W. 1978. Simultaneous measurement of nitrogen fixation estimated by acetylene-ethylene assay and nitrate absorption by soybeans. Plant Physiol. 62:443-448.

Xie, X., Huang, W., Liu, F., Tang, N., Liu, Y., Lin, H., and Zhao, B. 2013. Functional analysis of the novel mycorrhiza-specific phosphate transporter AsPT1 and PHT1 family from Astragalus sinicus during the arbuscular mycorrhizal symbiosis. New Phytol. 198:836-852.

Ye, R., Zhou, F., and Lin, Y. 2012. Two novel positive cis-regulatory elements involved in green tissue-specific promoter activity in rice (Oryza sativa L ssp.). Plant Cell Rep. 31:1159-1172.
Young, N. D., Debellé, F., Oldroyd, G. E., Geurts, R., Cannon, S. B. Udvardi, M. K., Benedito, V. A., Mayer, K. F., Gouzy, J., Schoof, H., Van de Peer, Y., Proost, S., Cook, D. R., Meyers, B. C., Spannagl, M. Cheung, F., De Mita, S., Krishnakumar, V., Gundlach, H., Zhou, S., Mudge, J., Bharti, A. K., Murray, J. D., Naoumkina, M. A., Rosen, B., Silverstein, K. A., Tang, H., Rombauts, S., Zhao, P. X., Zhou, P., Barbe, V., Bardou, P., Bechner, M., Bellec, A., Berger, A., Bergès, H., Bidwell, S., Bisseling, T., Choisne, N., Couloux, A., Denny, R., Deshpande, S., Dai, X., Doyle, J. J., Dudez, A. M., Farmer, A. D., Fouteau, S., Franken, C., Gibelin, C., Gish, J., Goldstein, S., González, A. J., Green, P. J., Hallab, A., Hartog, M., Hua, A., Humphray, S. J., Jeong, D. H., Jing, Y., Jöcker, A., Kenton, S. M., Kim, D. J., Klee, K., Lai, H., Lang, C., Lin, S., Macmil, S. L., Magdelenat, G., Matthews, L., McCorrison, J., Monaghan, E. L., Mun, J. H., Najar, F. Z., Nicholson, C., Noirot, C., O’Bleness, M., Paule, C. R., Poulain, J., Prion, F., Qin, B., Qu, C., Retzel, E. F., Riddle, C., Sallet, E., Samain, S., Samson, N., Sanders, I., Saurat, O., Scarpelli, C., Schiex, T., Segurens, B., Severin, A. J., Sherrier, D. J., Shi, R., Sims, S., Singer, S. R., Sinharoy, S., Sterck, L., Viollet, A., Wang, B. B., Wang, K., Wang, M., Wang, X., Warfsmann, J., Weissenbach, J., White, D. D., White, J. D., Wiley, G. B., Wincker, P., Xing, Y., Yang, L., Yao, Z., Ying, F., Zhai, J., Zhou, L., Zuber, A., Dénarié, J., Dixon, R. A., May, G. D., Schwartz, D. C., Rogers, J., Quétier, F., Town, C. D., and Roe, B. A. 2011. The Medicago genome provides insight into the evolution of rhizobial symbioses. Nature 480: 520-524. 